\title{
Probing the ejecta of evolved massive stars in transition
}

\section{A VLT/SINFONI $K$-band survey $\star, \star \star$}

\author{
M. E. Oksala ${ }^{1}$, M. Kraus ${ }^{1}$, L. S. Cidale ${ }^{2,3}$, M. F. Muratore ${ }^{2,3}$, and M. Borges Fernandes ${ }^{4}$ \\ 1 Astronomický ústav, Akademie věd České republiky, Fričova 298, 25165 Ondřejov, Czech Republic \\ e-mail: oksala@sunstel.asu.cas.cz \\ 2 Departamento de Espectroscopía Estelar, Facultad de Ciencias Astronómicas y Geofísicas, \\ Universidad Nacional de La Plata (UNLP), Paseo del Bosque S/N, 1900 La Plata, Argentina \\ 3 Instituto de Astrofísica La Plata, CCT La Plata, CONICET, Paseo del Bosque S/N, 1900 La Plata, Argentina \\ ${ }^{4}$ Observatório Nacional, Rua General José Cristino, 77 São Cristovão, 20921-400 Rio de Janeiro, Brazil
}

Received 25 March 2013 / Accepted 2 August 2013

\begin{abstract}
Massive evolved stars in transition phases, such as luminous blue variables (LBVs), B[e] supergiants (B[e]SGs), and yellow hypergiants (YHGs), are not well understood, and yet crucial steps in determining accurate stellar and galactic evolution models. The circumstellar environments of these stars reveal their mass-loss history, identifying clues to both their individual evolutionary status and the connection between objects of different phases. Here we present a survey of 25 such evolved massive stars (16 B[e]SGs, 6 LBVs, 2 YHGs, and 1 Peculiar Oe star), observed in the $K$-band with the Spectrograph for INtegral Field Observation in the Near-Infrared (SINFONI; $R=4500$ ) on the ESO VLT UT4 $8 \mathrm{~m}$ telescope. The sample can be split into two categories based on spectral morphology: one group includes all of the B[e]SGs, the Peculiar Oe star, and two of the LBVs, while the other includes the YHGs and the rest of the LBVs. The difference in LBV spectral appearance is due to some objects being in a quiescent phase and some objects being in an active or outburst phase. $\mathrm{CO}$ emission features are found in 13 of our targets, with first time detections for MWC 137, LHA 120-S 35, and LHA 115-S 65. From model fits to the CO band heads, the emitting regions appear to be detached from the stellar surface. Each star with ${ }^{12} \mathrm{CO}$ features also shows ${ }^{13} \mathrm{CO}$ emission, signaling an evolved nature. Based on the level of ${ }^{13} \mathrm{C}$ enrichment, we conclude that many of the B[e]SGs are likely in a pre-Red Supergiant phase of their evolution. There appears to be a lower luminosity limit of $\log L / L_{\odot}=5.0$ below which $\mathrm{CO}$ is not detected. The lack of CO features in several high luminosity $\mathrm{B}$ [e]SGs and variability in others suggests that they may in fact be LBV candidates, strengthening the connection between these two very similar transition phases.
\end{abstract}

Key words. infrared: stars - techniques: spectroscopic - stars: massive - circumstellar matter - stars: evolution

\section{Introduction}

The post-main sequence evolution of massive $\left(M_{\text {ini }} \geq 8 M_{\odot}\right)$ stars remains one of the most difficult and confusing problems facing both observation and theory. Although there are well behaved evolved stars, following theoretical prescribed paths through the supergiant phases, much interest has been paid to the more perplexing transition stages. The path from the end of the main sequence through to the point of supernova can be substantially different for stars of varying mass, metallicity, rotation, etc. (see e.g., Maeder \& Meynet 2004; Meynet et al. 2011), which can result in inaccurate stellar evolutionary model predictions, and as a result inadequate galactic evolution models. During the transition phases, large amounts of mass are released from the stellar surface by strong stellar winds and/or eruption events. The ejected material is detected in the circumstellar environment as shells, rings, or disks, and conditions often can facilitate the formation of molecules and dust. The mechanism triggering such mass ejections (i.e. rapid rotation, pulsation, other instabilities) is not well known and may be different for various phases.

* Based on observations at the European Southern Observatory, Paranal, under program IDs 384.D-1078(A) and 088.D-0442(B).

$\star \star$ Tables 3-5, and Fig. 6 are available in electronic form at http://www . aanda.org
Further complicating matters is the lack of information given in the optical spectra of these stars. Often, the circumstellar material masks the photospheric contribution with strong permitted and forbidden emission, making stellar parameters difficult to obtain.

Many of the transition phases, specifically luminous blue variables (LBVs), B[e] supergiants (B[e]SGs), and yellow hypergiants (YHGs), are poorly understood and still remain quite puzzling. LBVs expel large amounts of mass via eruptions likely arising as the star encroaches the Eddington luminosity limit (for reviews see Humphreys \& Davidson 1994; Nota \& Lamers 1997). These stars exhibit a wide variety of physical signatures of this mass-loss, with observational variability presenting as their primary characteristic. LBVs become redder as they brighten, due to the simultaneous expansion and cooling of the photosphere as the star transitions visually from a hot supergiant in its quiescence (visual minimum) phase to a cool supergiant during its outburst (visual maximum) period. This sequence is referred to as the S-Dor cycle of LBVs and repeats on the timescale of months to years. The expansion of the photosphere is also referred to as the formation of a "pseudo-photosphere", as described by Leitherer et al. (1985). Occasionally, an LBV may experience a more intense, longer mass-loss period, or large eruption (e.g., $\eta$ Carinae, HD 5980). This type of event 
typically occurs on timescales of a thousand years. The progenitors of LBVs are very massive, very luminous stars, however the physical state of these stars prior to or post LBV classification is unknown. $\mathrm{B}[\mathrm{e}] \mathrm{SG}$ show various observational signatures of a dense circumstellar disk or ring, including narrow forbidden emission lines, molecular emission, and evidence for dust (for a general review see Lamers et al. 1998). Zickgraf et al. (1985) proposed a disk formation mechanism for B[e] stars, in which rapid rotation produces a hybrid wind consisting of a fast line-driven polar component and a slow, high density equatorial component (with further study by, e.g., Lamers \& Pauldrach 1991; Pelupessy et al. 2000; Kraus \& Lamers 2003; Curé 2004; Curé \& Rial 2004; Curé et al. 2005; Kraus 2006). While the spectral features certainly support this process, rotational speeds have been determined for only a few B[e]SGs including LHA 115-S 23 (Kraus et al. 2008), LHA 115-S 65 (Zickgraf 2000; Kraus et al. 2010), LHA 120-S 93 (Gummersbach et al. 1995), and LHA 120-S 73 (Zickgraf 2006). Moreover, several recent studies show that this mechanism may not be a feasible explanation, based on the detection of detached rings/disks (Kraus et al. 2010; Kastner et al. 2010; Liermann et al. 2010). YHGs are suggested to be cool, post-Red Supergiant (post-RSG) objects surrounded by dense shells of material expelled, presumably, by pulsational instabilities (de Jager 1998). These objects could be possible progenitors of LBVs and/or B[e]SGs, although their involvement in the evolution of massive stars remains unclear. To make the evolutionary picture even more ambiguous, each of these distinct stages occupy similar space in the HertzsprungRussell diagram (HRD), as shown in Fig. 4 of Aret et al. (2012). Currently, stellar evolution codes do not predict any of these transition phases.

In the circumstellar regions of massive stars, strong radiation-driven stellar winds should prevent the formation of molecules and dust. However, in a disk structure, $\mathrm{CO}$ molecules can shield themselves from the destructive stellar radiation field, and attain a high enough density for CO molecular emission to be observed. The seminal work of McGregor et al. (1988a,b, 1989) confirmed this for certain evolved emission line stars. The existence of $\mathrm{CO}$ band head emission is only possible if certain physical features of the material are met. At warm temperatures $(\sim 2000-5000 \mathrm{~K})$ and high densities $\left(>10^{10} \mathrm{~cm}^{-3}\right)$, molecules are predicted to be abundant and sufficiently excited to generate spectral band head features. These types of conditions are also found in the circumstellar environments of pre-main sequence objects such as Herbig Ae/Be stars or Herbig Ae/B[e] stars, which makes it sometimes difficult to assign a star the proper evolutionary stage.

To distinguish the true evolutionary stage of these stars, Kraus (2009) and Liermann et al. (2010) have demonstrated that $\mathrm{CO}$ emission features are not only useful to evaluate the physical conditions of the circumstellar material, but also to determine the presence of enrichment of the carbon isotope ${ }^{13} \mathrm{C}$, brought to the surface as a consequence of chemical reactions in the core and mixing processes. In the interstellar medium, the ratio of ${ }^{12} \mathrm{C}$ to ${ }^{13} \mathrm{C}$ is approximately 90 , which is the value used as the initial ratio in stellar evolution calculations (e.g., Ekström et al. 2012). By the end of the star's main sequence lifetime it should have decreased to 20 (as discussed in Sect. 4.1.2). Because many of these stars are shrouded by their circumstellar environment, it would be difficult to determine this measurement. However, if the ejected material is a product of post-main sequence events (Kraus 2009), the circumstellar matter seen in the spectrum of these stars should accurately reflect the composition of the stellar surface and can be used as a diagnostic tool.
In this paper, we present the results of our medium resolution ( $R=4500) K$-band survey of evolved massive stars: B[e]SGs, LBVs, YHGs and a Peculiar Oe star, to extract information about their circumstellar material, and to determine their possible evolutionary connections. Section 2 presents the sample, the observations, and the data reduction process. Additionally, this section presents the observed spectral morphology results. Spectral models are explained and results reported in Sect. 3. In Sect. 4, we discuss the specific outcomes of this study, and their possible implications for understanding massive star evolution. Section 5 summarizes our conclusions and states suggestions for future work.

\section{Observations and spectral morphology}

\subsection{Sample selection}

We obtained $K$-band spectroscopic data for a near-infrared study of the molecular circumstellar material and possible evolutionary links between various phases of massive star evolution. The target stars in this sample were chosen to highlight possible physical stages of massive star evolution that may be connected, with an emphasis on the group of particularly perplexing B[e]SGs. The sample contains 25 massive evolved stars in the Galaxy and the Magellanic Clouds, including $16 \mathrm{~B}[\mathrm{e}] \mathrm{SGs}$, 6 LBVs, 2 YHGs, and 1 Peculiar Oe star. Each target star is listed in Table 1 together with various properties and stellar parameters derived from the literature. In the case of LHA 115-S 6, we consider it a B[e]SG (Zickgraf et al. 1987), although it has shown characteristics typical of an LBV (Zickgraf et al. 1996). Included in this study are all of the known Magellanic Cloud B[e]SGs studied by Zickgraf et al. (1986), with the exception of LHA 120-S 111 in the Large Magellanic Cloud (LMC) and LHA 115-S 23 in the Small Magellanic Cloud (SMC). These two stars were excluded because of their faint magnitudes. The chosen targets represent not only distinct evolutionary stages, but also a variety of initial masses, luminosities, and effective temperatures. Many of the objects have $K$-band spectra featured in the works of McGregor et al. (1988a,b, 1989), including the first detection of $\mathrm{CO}$ emission in an evolved massive star (McGregor et al. 1988a), although for several objects this is the first observation of this wavelength region.

\subsection{Data acquisition and reduction}

For each sample target, a medium resolution $(R=4500) K$-band spectrum was obtained with the Spectrograph for INtegral Field Observation in the Near-Infrared (SINFONI; Eisenhauer et al. 2003; Bonnet et al. 2004), on the ESO VLT UT4 8 m telescope. The observations were obtained from 2009-2012 (individual dates of observation and exposure times are listed in Table 2). The spectrograph was set to an $8 \times 8 \operatorname{arcsec}^{2}$ field of view (corresponding to a 250 mas spatial resolution). The observations were taken in an ABBA nod pattern for optimal sky subtraction. A B-type standard star was observed immediately following each target star and at a similar airmass value to perform both telluric correction and flux calibration.

The raw data for both the target and standard stars were reduced using the SINFONI pipeline (version 2.2.9). The observations were treated for bad pixels, flat fields, and distortions, and then wavelength calibrated. In the infrared, there can be a large amount of telluric lines that can be difficult to remove. We chose to use main sequence B-type standards, since these stars have few spectral features in the infrared wavelength region, typically 
M. E. Oksala et al.: Probing the ejecta of evolved massive stars in transition

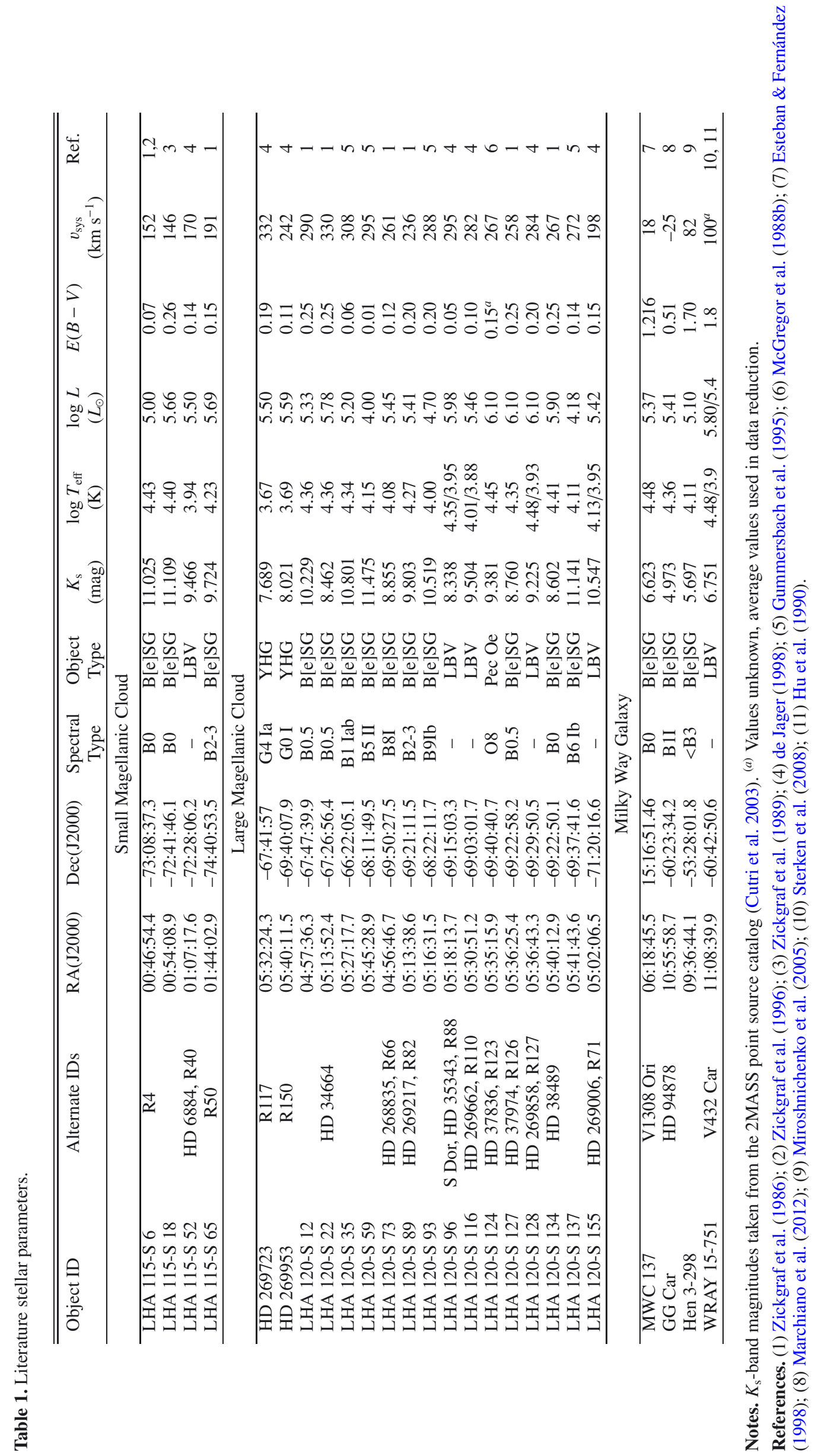

A17, page 3 of 20 
Table 2. Observation summary.

\begin{tabular}{|c|c|c|c|c|c|}
\hline Object & Date & $\begin{array}{l}\text { Time } \\
\text { UT }\end{array}$ & $\begin{array}{c}t_{\mathrm{EXP}} \\
(\mathrm{s})\end{array}$ & $\begin{array}{c}\text { Final } \\
S / N\end{array}$ & $\begin{array}{c}K_{\mathrm{s}} \\
(\mathrm{mag})\end{array}$ \\
\hline \multirow[t]{2}{*}{ LHA 115-S 6} & 2011-10-05 & $01: 48: 43$ & 150 & 200 & 11.18 \\
\hline & 2011-10-06 & 04:09:34 & 150 & - & - \\
\hline \multirow[t]{2}{*}{ LHA 115-S 18} & 2009-10-12 & $01: 56: 49$ & 300 & 100 & 10.90 \\
\hline & $2009-10-16$ & $02: 27: 33$ & 300 & - & - \\
\hline LHA 115 -S 52 & 2011-10-06 & 04:55:07 & 30 & 150 & 8.77 \\
\hline LHA $115-\mathrm{S} 65$ & 2011-10-06 & $05: 26: 58$ & 60 & 200 & 9.63 \\
\hline HD 269723 & $2011-12-23$ & $00: 31: 35$ & 10 & 150 & 7.69 \\
\hline HD 269953 & 2012-01-21 & 03:46:10 & 10 & 150 & 8.15 \\
\hline LHA 120-S 12 & 2009-10-14 & $07: 55: 34$ & 150 & 150 & 11.36 \\
\hline LHA 120-S 22 & 2011-12-23 & $00: 31: 35$ & 20 & 150 & 8.73 \\
\hline \multirow[t]{2}{*}{ LHA 120-S 35} & 2012-02-16 & $00: 18: 47$ & 150 & 200 & 11.33 \\
\hline & $2012-02-16$ & $02: 43: 18$ & 150 & - & - \\
\hline \multirow{3}{*}{ LHA 120-S 59} & 2011-11-20 & $07: 38: 41$ & 150 & 250 & 11.60 \\
\hline & 2012-01-10 & 05:13:49 & 150 & - & - \\
\hline & 2012-02-04 & 01:10:49 & 150 & - & - \\
\hline LHA $120-S 73$ & 2009-11-09 & 07:09:21 & 100 & 200 & 9.10 \\
\hline LHA $120-\mathrm{S} 89$ & 2012-02-01 & $00: 58: 53$ & 60 & 300 & 10.03 \\
\hline LHA 120-S 93 & $2012-01-26$ & $02: 40: 25$ & 150 & 100 & 10.19 \\
\hline LHA 120-S 96 & 2011-10-11 & $08: 35: 59$ & 20 & 150 & 9.13 \\
\hline LHA 120-S 116 & 2012-02-04 & 02:05:39 & 60 & 150 & 9.74 \\
\hline LHA 120-S 124 & 2012-02-04 & $02: 28: 10$ & 60 & 100 & 9.60 \\
\hline LHA 120-S 127 & 2012-02-05 & $01: 21: 52$ & 30 & 200 & 8.72 \\
\hline LHA 120-S 128 & 2012-02-05 & $01: 33: 10$ & 30 & 150 & 9.28 \\
\hline LHA 120-S 134 & 2009-11-10 & $08: 25: 07$ & 30 & 150 & 8.43 \\
\hline \multirow[t]{2}{*}{ LHA 120-S 137} & 2012-01-21 & $03: 46: 10$ & 150 & 300 & 11.78 \\
\hline & 2012-01-21 & $04: 05: 55$ & 150 & - & - \\
\hline LHA 120-S 155 & 2012-01-26 & 01:47:05 & 60 & 100 & 7.09 \\
\hline MWC 137 & 2011-10-05 & $08: 46: 49$ & 2 & 200 & 6.68 \\
\hline GG Car & 2012-01-09 & $05: 36: 17$ & 0.83 & 60 & 5.55 \\
\hline Hen 3-298 & 2012-01-09 & $05: 55: 56$ & 1 & 200 & 5.56 \\
\hline WRAY 15-751 & 2012-01-08 & 08:00:00 & 1 & 200 & 4.16 \\
\hline
\end{tabular}

Notes. 2MASS $K_{\mathrm{s}}$-band magnitudes derived from the flux calibrated spectrum.

lines of hydrogen and helium. Using these standard spectra, we created telluric templates and corrected each target spectrum using the $\mathrm{IRAF}^{1}$ task telluric. Some areas of telluric contamination were particularly difficult to correct, leaving remnant traces in these specific wavelength regions. To flux calibrate each target spectrum, the corresponding standard star spectrum was scaled with an appropriate Kurucz flux model (Kurucz 1993) to its Two Micron All-Sky Survey (2MASS; Skrutskie et al. 2006) $K_{\mathrm{s}}$-band magnitude.

The resultant spectra were then corrected (using the values listed in Table 1) for heliocentric and systemic velocities and dereddened with the appropriate corresponding $E(B-V)$ values, according to the interstellar extinction relation of Howarth (1983) assuming $R=3.1$. The final spectra have signal-tonoise ratios (S/N) ranging from 60-300 (listed in Table 2). Figures 1-4 show the full spectrum for each of our targets. The $K$-band of the SINFONI spectrograph has a wavelength range of 1.95-2.45 $\mu \mathrm{m}$, containing the Brackett hydrogen recombination line $(\mathrm{Br} \gamma)$ at $2.167 \mu \mathrm{m}$, the upper levels of the Pfund series, and various helium and metal lines. Additionally, the first overtone band heads for the $\mathrm{CO}$ molecule are located within this region.

\footnotetext{
1 IRAF is distributed by the National Optical Astronomy Observatory, which is operated by the Association of Universities for Research in Astronomy (AURA) under cooperative agreement with the National Science Foundation.
}

\subsection{K-band magnitudes}

From the flux calibrated final spectra, we computed the 2MASS $K_{\mathrm{S}}(K$ short $)$ magnitude at the time of observation for each target. The calculation utilized the filter function and calibration constant (zero point flux) given by Cutri et al. (2003), as described in Liermann et al. (2009). These values are listed in the last column of Table 2 . The process of obtaining flux calibrated spectra contains uncertainties, such as stability of the magnitude and the effective temperature assumed for the standard star. Also, the telluric correction process can influence the flux level. Comparison between the 2MASS $K_{\mathrm{s}}$-band measurements and these newly determined values indicate that in most cases the two values are similar with differences typically below $\sim 0.5 \mathrm{mag}$, likely due to the aforementioned uncertainties. In several cases though, the change in magnitude appears significant. The LBVs LHA 115S 52, Wray 15-751, and LHA 120-S 155 all show brighter magnitudes, with the latter two significantly increased by 2.6 and $3.5 \mathrm{mag}$, respectively. The LBV LHA 120-S 96 (S Dor) shows a slight decrease in brightness. The B[e]SG LHA 120-S 12 is the only non-LBV that shows notable magnitude change, with a decrease in brightness of approximately one magnitude.

\subsection{Continua}

To properly model the pure $\mathrm{CO}$ emission, we subtracted a continuum from the spectrum (described in Sect. 3, fit using the portion of the spectrum from $\sim 2.17-2.25 \mu \mathrm{m}$ ). While the area of interest for the modeling procedure is only a small section of the spectrum, the "normalization" of each spectra produced two distinct groups (i) kinked and (ii) generally flat spectra, based solely on the shape of their continua.

Stars within the first group display a shape of the continuum featuring two bends or kinks, one at $\sim 2.1 \mu \mathrm{m}$ and another at $\sim 2.3 \mu \mathrm{m}$. Such a behavior is already noticeable for one object, the YHG HD 269953 (see Fig. 5) upon visual inspection of its flux calibrated spectrum without normalization. Because of the broad range of flux in Figs. 1-4, other objects that fall into this category became clear only after normalization. The remaining members of this group are the second YHG star, HD 269723, and the LBVs LHA 115-S 52, LHA 120-S 116, LHA 120-S 155, and WRAY 15-751. The spectrum in all these stars slopes upward blue-ward of the first bend and slopes upward again redward of the second. The slope in the red portion of the spectrum may be due to the presence of dust in the circumstellar environment, while the blue ward increasing slope indicates a cool stellar photospheric spectrum in all these objects (see Sect 4.1.1). The location of the bends depend on the physical conditions of the dust, the strength of the stellar wind, and the temperature of the star. To highlight this kinked shape, Fig. 5 shows continuum subtracted spectra of two examples, the YHG HD 269953 and the LBV WRAY 15-751. Figure 6 contains a plot showing all six of the objects of this group.

In the other group of objects, the continuum throughout the entire $K$-band wavelength range is generally flat. Several target spectra do have an increasing slope in the red most portion of the spectrum in the location of the CO band heads and/or Pfund lines, due to the presence of a hot dust continuum. The objects in this group include all of the B[e]SGs, the Peculiar Oe star LHA 120-S 124, and the LBVs LHA 120-S 96 (S Dor) and LHA 120-S 128. Two examples of this second group of objects are shown in Fig. 7, the B[e]SG LHA 120-S 22 and the LBV S Dor. As the figure shows, visually the B[e]SGs and these LBVs are indistinguishable. 
M. E. Oksala et al.: Probing the ejecta of evolved massive stars in transition
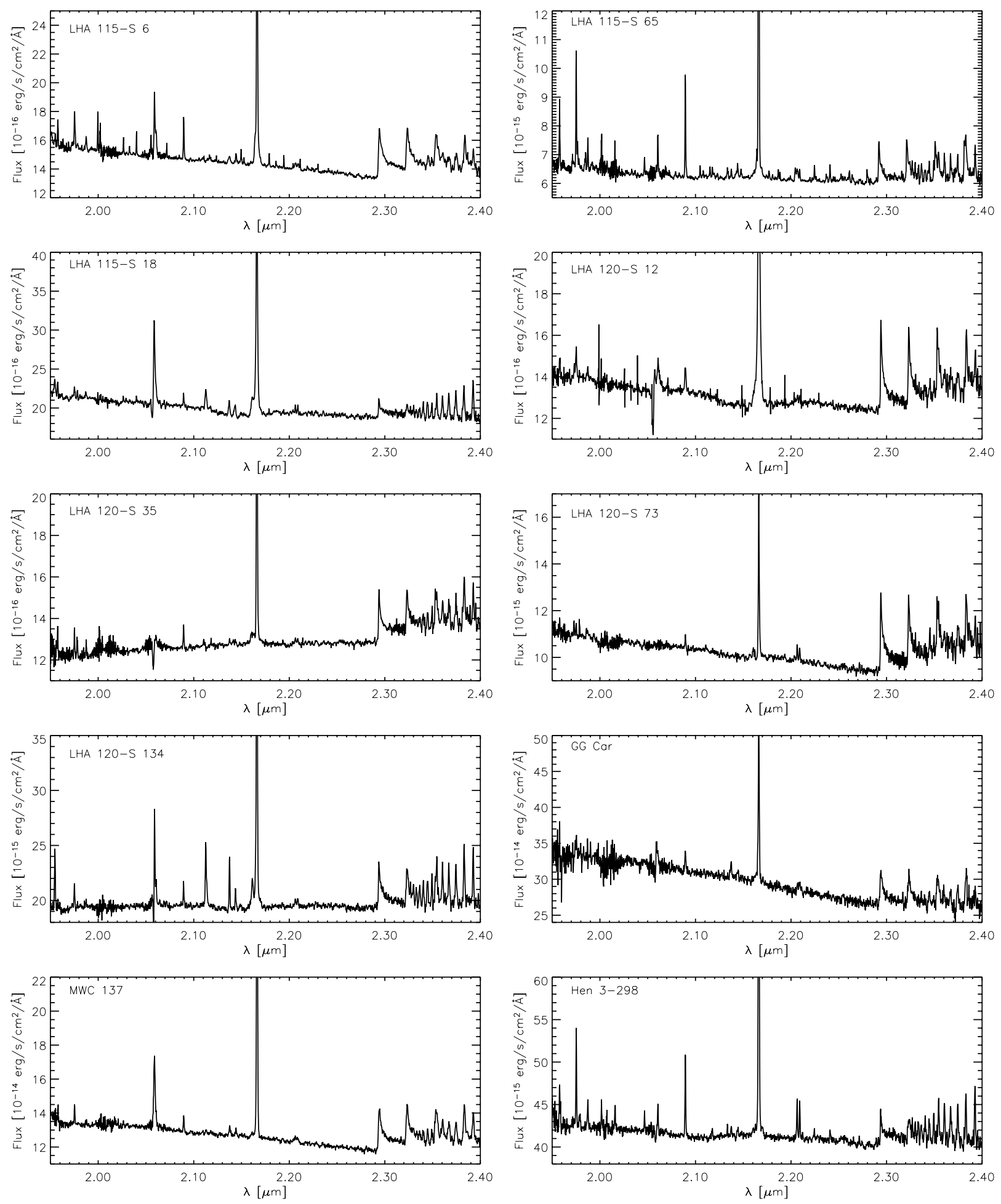

Fig. 1. Flux-calibrated SINFONI $K$-band spectra of B[e]SGs with definite CO band head emission.

\subsection{Spectral composition}

The objects in our survey show a variety of spectral features, with some spectra containing a large number of emission lines, while others only contain a few absorption lines. Each spectrum has been analyzed, and Tables 3-5 contain line identifications and equivalent width measurements for each target star. Each of the objects in our survey shows evidence of the $\operatorname{Br} \gamma$ line in either emission or absorption. Because of a close blend with several He I lines, it is difficult to determine the proper shape of the $\mathrm{Br} \gamma$.
In fact, with the exception of LHA 120-S 124, the Br $\gamma$ line is the strongest feature in the spectrum. All B[e]SGs except LHA 120$\mathrm{S} 93$ show Pfund lines in emission, as well as the Peculiar Oe star LHA 120-S 124. The LBVs S Dor and LHA 120-S 128 both show Pfund emission, while LHA 115-S 52, LHA 120-S 116, LHA 120-S 155, and WRAY-15 751 show these lines in absorption. Neither of the two YHG stars show evidence of Pfund line features.

Examining the spectral features of our collective survey, the groups discussed in the previous section, separated according 

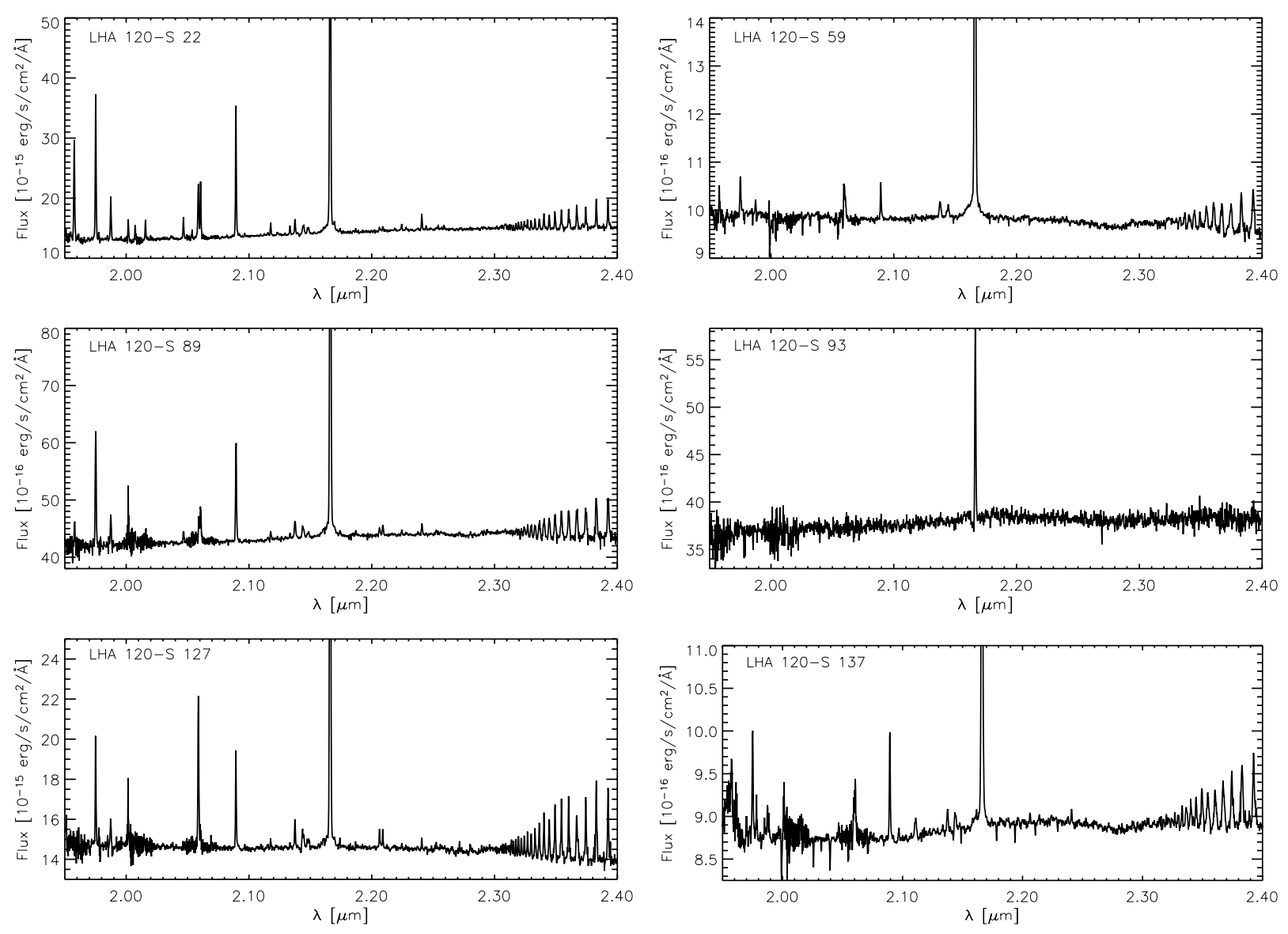

Fig. 2. Flux-calibrated SINFONI $K$-band spectra of B[e]SGs with absent or uncertain CO band head emission.

to continua shape, also correspond to distinct spectral compositions. Stars in the first group, characterized by a kinked continuum, contain few, primarily absorption lines in their spectra, including lines of H I, Na I, and Mg II, typical of cooler (A-, F-, or G-type) supergiants. Spectra in the second group, characterized by a generally flat continuum, contain a large number of emission lines (e.g., H I, He I, Fe II, [Fe II], Mg II, Na I, and/or Ca I), with some targets showing lines in absorption as well. These spectra are more representative of hotter (O- or B-type) supergiants. LHA 120-S 12 shows in several of its spectral features what appear to be shell line profiles, possibly due to its edge-on orientation (Aret et al. 2012). In several objects, helium lines are found in absorption, consistent with a B-type stellar spectrum.

Out of the 25 sample stars, we detected CO band head emission in a total of 13 objects, of which 10 are classified as B[e]SGs, 2 as YHGs, and 1 as a Peculiar Oe star (see Fig. 8). For three of these stars, LHA 115-S 65 (Oksala et al. 2012), MWC 137 (Muratore et al. 2013) and LHA 120-S 35 (Torres et al., in prep.), this study is the initial detection of $\mathrm{CO}$ emission. The remaining 10 stars are confirmed detections, previously known to show CO in emission. The spectrum of LHA 120-S 59 shows evidence for weak $\mathrm{CO}$ emission, although the contamination of strong Pfund emission makes this determination unclear. If present, it would be the first detection of $\mathrm{CO}$ in this star. We find that none of the LBVs show evidence of $\mathrm{CO}$ molecular emission. Although our sample is small, it seems that the lower metallicity of the SMC compared to Galactic metallicity does not seem to have an effect on the presence of $\mathrm{CO}$ emission. However, all three of the observed SMC B[e]SGs from this sample are highly luminous, and we cannot rule out an observational bias. It is interesting though to note that there does appear to be a lower luminosity limit, with no stars lower than $\log L / L_{\odot}=5.0$ showing definitive molecular band head emission. Of the LMC $\mathrm{B}$ [e]SGs, we find 6 of the 10 do not show $\mathrm{CO}$ emission, three of which are below this "luminosity limit" (LHA 120-S 59, LHA 120-S 93, and LHA 120-137).

\section{Spectral modeling}

Visual inspection of the region between 2.27 and $2.4 \mu \mathrm{m}$ reveals that many of the observed objects contain evidence of circumstellar material either in the form of hydrogen recombination emission lines from the Pfund series, or first overtone band head emission from $\mathrm{CO}$ molecules. To determine the physical parameters of the $\mathrm{CO}$ emitting region, the contribution from other sources was removed. Using the spectral region between the $\mathrm{Br} \gamma$ line and the start of the $\mathrm{CO}$ band heads, we determined a suitable linear fit to the continuum using the IRAF task continuum for each target. This linear fit was then subtracted from the spectrum to determine the accurate $\mathrm{CO}$ contribution to the flux. This spectrum was compared with synthetic spectra to determine the best fit model, using the codes described in the following sections. We find a variety of modeling situations, with some spectra requiring both $\mathrm{CO}$ and Pfund models, while some only require one or the other. When only Pfund emission lines are visibly present, the spectra were modeled accordingly, and inspected for any trace of $\mathrm{CO}$ emission. In the case of no spectral features or Pfund lines in absorption, we do not compute a model. 
M. E. Oksala et al.: Probing the ejecta of evolved massive stars in transition
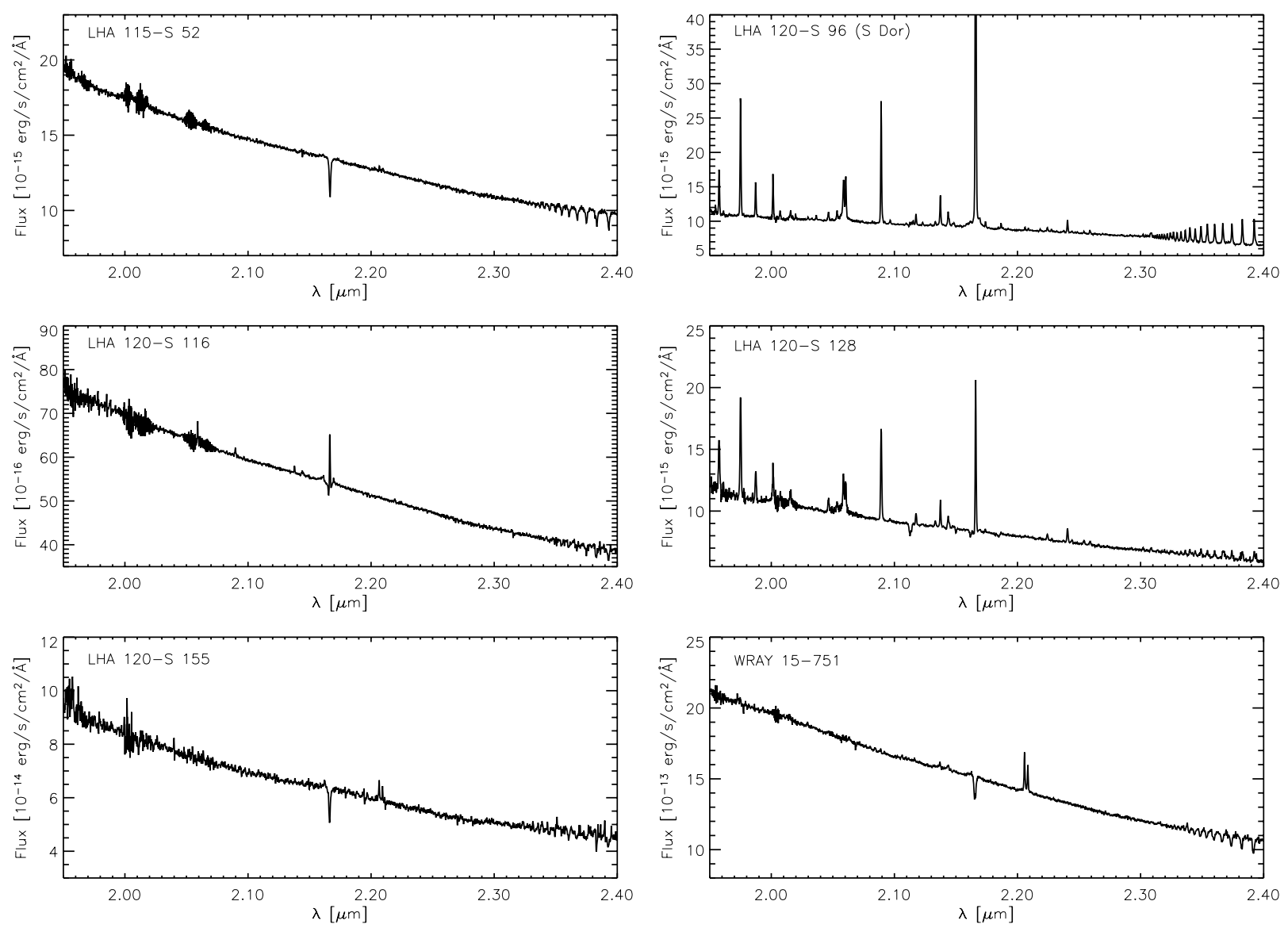

Fig. 3. Flux-calibrated SINFONI $K$-band spectra of LBVs.

\subsection{Pfund model}

The Pfund series emission spectrum was in most cases modeled with the code developed by Kraus et al. (2000), with the spectral lines computed using a simple Gaussian profile. The emission lines are assumed to be optically thin and in LTE. This model is applicable in the case that the emitting material originates in a wind or a shell. In a few cases, we noted that the observed Pfund emission lines were not well fit by a Gaussian and appeared to be double peaked. We therefore developed a new code, similar to the CO emission code of Kraus et al. (2000), to consider emitting material located in a narrow ring within a rotating disk.

\subsection{CO model}

For the model computations of $\mathrm{CO}$ band head emission, we utilized the previously developed code of Kraus et al. (2000), which computes a ${ }^{12} \mathrm{CO}$ emission spectrum with the material located within a small ring in a rotating disk with lines broadened by a rotational velocity $v_{\text {rot }}$, and the code of Kraus (2009) which computes a non-rotating spectrum of both ${ }^{12} \mathrm{CO}$ and ${ }^{13} \mathrm{CO}$ band head emission. In both cases the $\mathrm{CO}$ gas is assumed to be in LTE. Since several of the objects with CO emission appeared rotationally broadened (beyond the SINFONI velocity resolution of $60 \mathrm{~km} \mathrm{~s}^{-1}$ ), but with significant ${ }^{13} \mathrm{CO}$ emission, we used the codes of Kraus et al. $(2000,2009)$ as the base for a hybrid model that computes both the ${ }^{12} \mathrm{CO}$ and ${ }^{13} \mathrm{CO}$ emission spectra assuming that the emitting material is located in a thin, rotating ring within the circumstellar environment. The assumption that the material is located in a relatively narrow ring around the star

with constant temperature, column density, and rotational velocity is supported by both theory (see e.g., Kraus 2009) and observations (see e.g., Kraus et al. 2000, 2013; Liermann et al. 2010). It may be possible that the material is distributed in a much different configuration, such as a shell or bipolar LBV type ejecta.

\subsection{Model results}

Model computations encompassed a large range of temperatures and column densities. The models have each been computed using the codes described in the previous sections, with only three stars (MWC 137, GG Car, and LHA 115-S 6) requiring an additional rotational broadening greater than the instrumental broadening of $60 \mathrm{~km} \mathrm{~s}^{-1}$. The best fit models to each of the targets with $\mathrm{CO}$ band head emission and/or Pfund emission are plotted in red in Figs. 9-11, except for HD 269723 (see the explanation below). The spectra shown in these figures have been continuum subtracted to determine the accurate $\mathrm{CO}$ contribution to the flux (as described in the beginning of this section). In the case of LHA 120-S 59 (Fig. 11), two models are presented, one with weak $\mathrm{CO}$ emission and one without. Both models fit the observations relatively well, however, the quality of the spectrum makes differentiation impossible.

Tables 6 and 7 give the model parameters for each of the $\mathrm{CO}$ emission fits. We do not include the model parameters for HD 269723 in this table, as the parameters could not be determined accurately. One possible reason for this difficulty may be that, as a YHG, the star might be surrounded by a cool molecular shell, rather than a disk. Also excluded is the model of LHA 120S 59 as it is merely suggestive at this point. The last column of 
Table 6. Best-fit CO model parameters.

\begin{tabular}{lcccc}
\hline \hline Object & $\begin{array}{c}T_{\mathrm{CO}} \\
(\mathrm{K})\end{array}$ & $\begin{array}{c}N_{\mathrm{CO}} \\
\left(10^{21} \mathrm{~cm}^{-2}\right)\end{array}$ & $\begin{array}{c}{ }^{12} \mathrm{C} /{ }^{13} \mathrm{C} \\
A_{\mathrm{CO} \cos i} \\
\left(\mathrm{AU}^{2}\right)\end{array}$ \\
\hline LHA 115-S 6 & $2200 \pm 200$ & $5 \pm 2$ & $12 \pm 2$ & $1.00 \pm 0.08$ \\
LHA 115-S 18 & $2000 \pm 200$ & $8 \pm 3$ & $20 \pm 5$ & $8.9 \pm 0.6$ \\
LHA 115-S 65 & $3200 \pm 300$ & $1.5 \pm 0.5$ & $20 \pm 5$ & $11.7 \pm 0.9$ \\
HD 269953 & $3000 \pm 200$ & $2 \pm 0.5$ & $10 \pm 1$ & $41.5 \pm 1.1$ \\
LHA 120-S 12 & $2800 \pm 500$ & $2.5 \pm 0.5$ & $20 \pm 2$ & $2.33 \pm 0.06$ \\
LHA 120-S 35 & $3000 \pm 200$ & $2 \pm 0.5$ & $10 \pm 2$ & $1.16 \pm 0.06$ \\
LHA 120-S 73 & $2800 \pm 500$ & $3.5 \pm 0.5$ & $9 \pm 1$ & $17.8 \pm 0.5$ \\
LHA 120-S 124 & $3000 \pm 400$ & $9.5 \pm 0.5$ & $20 \pm 5$ & $0.90 \pm 0.02$ \\
LHA 120-S 134 & $2200 \pm 200$ & $2 \pm 1$ & $15 \pm 2$ & $62 \pm 14$ \\
MWC 137 & $1900 \pm 200$ & $3 \pm 2$ & $25 \pm 5$ & $2.04 \pm 0.27$ \\
GG Car & $3200 \pm 200$ & $5 \pm 3$ & $15 \pm 5$ & $0.58 \pm 0.05$ \\
Hen 3-298 & $2000 \pm 200$ & $0.8 \pm 0.4$ & $20 \pm 5$ & $11 \pm 4$ \\
\hline
\end{tabular}
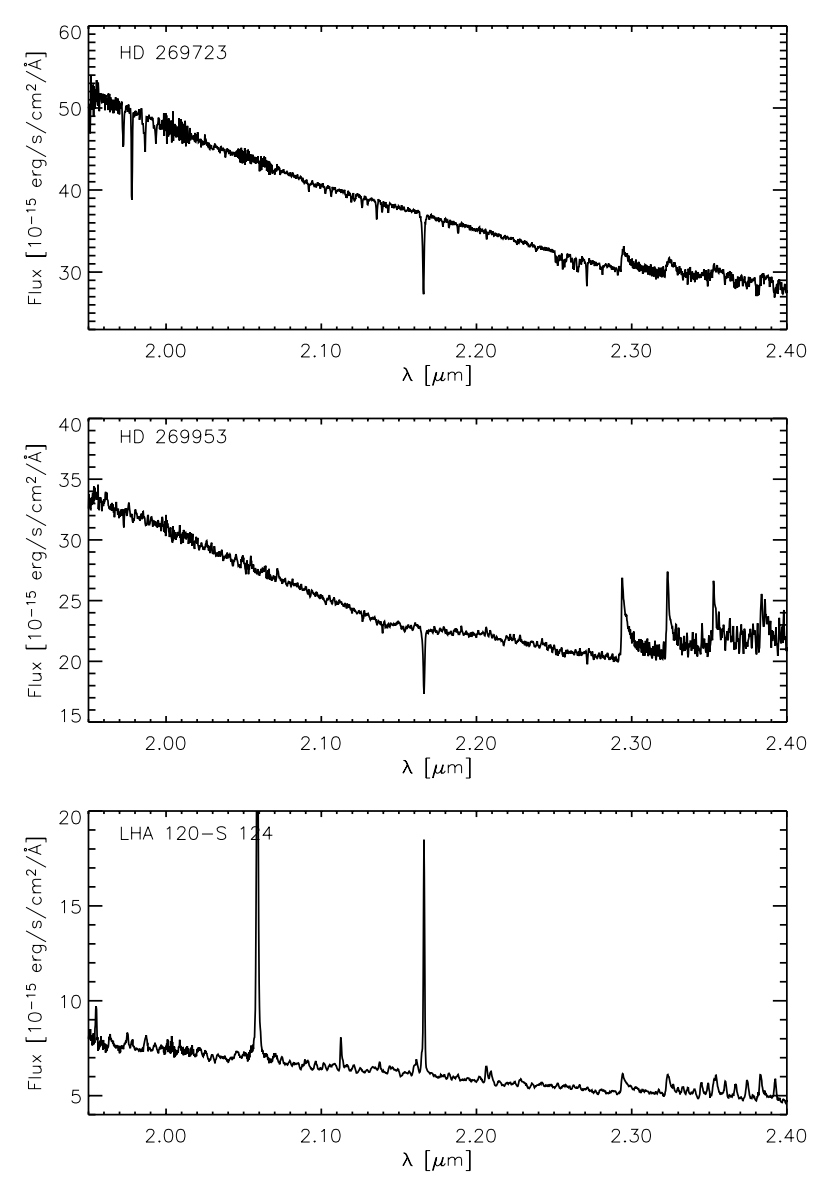

Fig. 4. Flux-calibrated SINFONI $K$-band spectra of the YHGs HD 269953 and HD 269723 and the Peculiar Oe star LHA 120-S 124.

Table 6 gives a line of sight computed area for the emitting ring of $\mathrm{CO}, \mathrm{A}_{\mathrm{CO}} \cos i$, in units of $\mathrm{AU}^{2}$. This quantity is derived from the ratio of the model fit to the flux calibrated spectrum. The area calculation assumes that each target's distance has been determined. For the LMC and SMC, these values are relatively well know at 48.5 and $61 \mathrm{kpc}$, respectively. The galactic objects have much less certainty in their distance determinations. For GG Car, Marchiano et al. (2012) determine a distance of $5 \pm 2 \mathrm{kpc}$. Esteban \& Fernández (1998) report a lower limit of $6 \mathrm{kpc}$ for the distance to MWC 137, assuming that the star is a supergiant. A range of 3-4.5 kpc for Hen 3-298 was reported
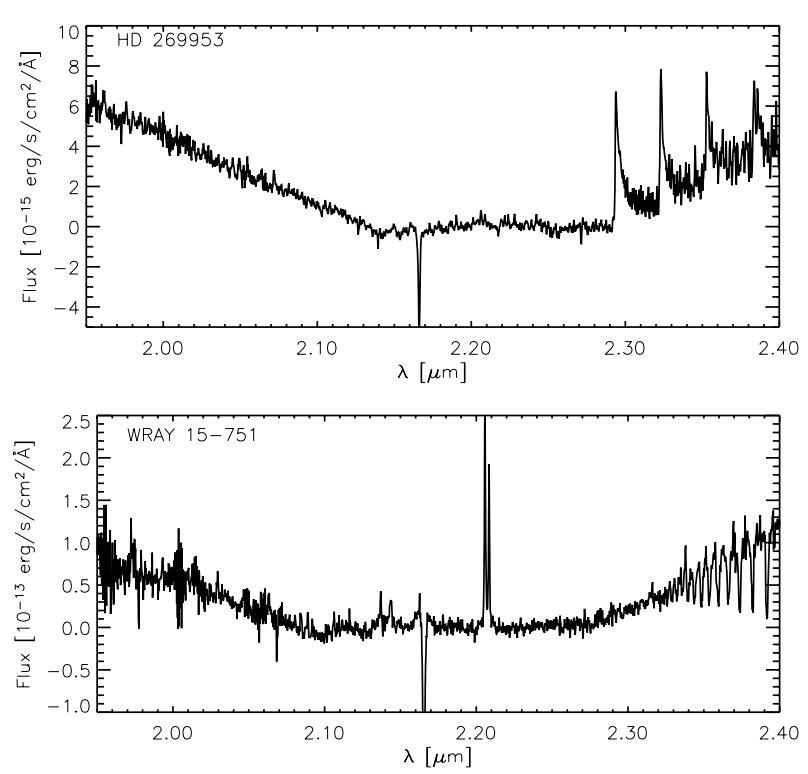

Fig. 5. Examples of the group of objects with cool supergiant type spectra. Flux-calibrated, continuum subtracted SINFONI $K$-band spectra of the YHG HD 269953 and the LBV WRAY 15-751.

by Miroshnichenko et al. (2005); we use the upper limit for our calculation, which results in an upper limit for the emitting area.

The objects in our survey with detected $\mathrm{CO}$ represent a range of $L$ and $T_{\text {eff }}$ values, however, there do not seem to be any correlations between stellar properties and the conditions in the circumstellar material where the $\mathrm{CO}$ molecules are formed. As $\mathrm{CO}$ band emission typically arises from the inner rim/edge of the molecular disk region, the fact that our $\mathrm{CO}$ model temperatures are all much cooler than the dissociation temperature of $5000 \mathrm{~K}$ indicates that the material may be located in a detached disk structure, as opposed to a disk that reaches the stellar surface as suggested by the model of Zickgraf et al. (1986). Such behavior has recently been reported in other studies (Kraus et al. 2010, 2013; Liermann et al. 2010; Wheelwright et al. 2012; Cidale et al. 2012).

Notably, one of the most engaging results from this study is that each of the targets with detectable ${ }^{12} \mathrm{CO}$ band head emission also shows clear signs of ${ }^{13} \mathrm{CO}$ emission. From the investigation by Kraus (2009), it is clear that pre-main sequence levels of ${ }^{13} \mathrm{C}$ should not produce visible molecular band head emission. We therefore conclude that each of the objects in this study with detected $\mathrm{CO}$ emission is in fact an evolved, post-main sequence 
M. E. Oksala et al.: Probing the ejecta of evolved massive stars in transition
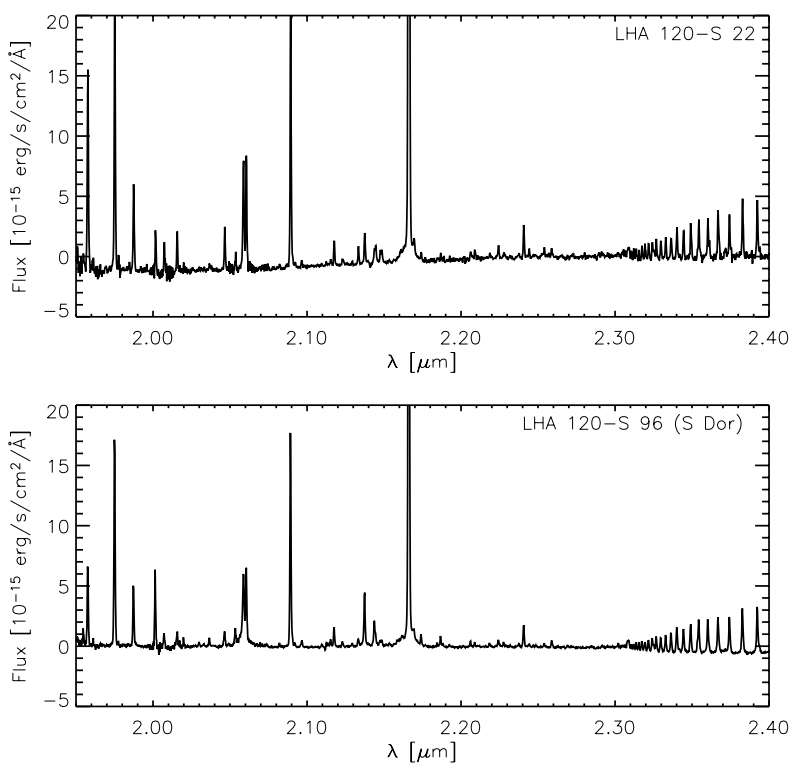

Fig. 7. Examples of the group of objects with hot supergiant type spectra. Flux-calibrated, continuum subtracted SINFONI $K$-band spectra of the B[e]SG LHA 120-S 22 and the LBV LHA 120-S 96 (S Dor).

Table 7. Broadening parameters used in model computation.

\begin{tabular}{|c|c|c|c|}
\hline Object & $\begin{array}{c}v_{\text {rot, CO }} \sin i \\
\left(\mathrm{~km} \mathrm{~s}^{-1}\right)\end{array}$ & $\begin{array}{c}v_{\text {rot,Pf }} \sin i \\
\left(\mathrm{~km} \mathrm{~s}^{-1}\right)\end{array}$ & $\begin{array}{c}v_{\text {Gauss,Pf } \sin i} i \\
\left(\mathrm{~km} \mathrm{~s}^{-1}\right)\end{array}$ \\
\hline LHA 115-S 6 & $100 \pm 10$ & $130 \pm 10$ & - \\
\hline MWC 137 & $85 \pm 10$ & - & $140 \pm 40$ \\
\hline $\mathrm{GG} \mathrm{Car}^{a}$ & $80 \pm 1$ & $94 \pm 2$ & - \\
\hline
\end{tabular}

Notes. Velocities determined by other work: ${ }^{(a)}$ Kraus et al. (2013) .

object. This point is especially poignant in the case of MWC 137 which has been frequently cited as a possible Herbig B[e] star (Hillenbrand et al. 1992).

\section{Discussion}

\subsection{Evolutionary status determination}

\subsubsection{LBVs}

Although our survey contains only a small number of LBV samples, it appears that there is clearly a dual dichotomy as far as spectral morphology is concerned. In fact, the overall continuum shape combined with the spectral line constitution is a clear sign of the physical state and the evolutionary status of such stars. If the spectrum appears with many emission lines and a relatively flat continuum, the star will be in a more blue ward position in the HRD, similar in appearance to the B[e]SGs. These LBVs are in their quiescent state and are not currently experiencing heavy or violent mass-loss events. Only two LBVs in our sample fall into this category, S Dor and LHA 120-S 128. In addition, these two stars happen to be the most luminous of our LBV sample, and are located near the Humphreys-Davidson limit in the HRD (see Fig. 8). Based on the classification scheme presented by Clark et al. (2011) for LBVs, the strong presence of He I $2.058 \mu \mathrm{m}$ indicates that both of these stars are quite hot.

The remaining LBVs in our sample that display the double kinked, YHG-like continuum with few, mostly absorption lines all have lower luminosities than their B[e]SG-like counterparts, and happen to appear in their cooler HRD position. The spectral

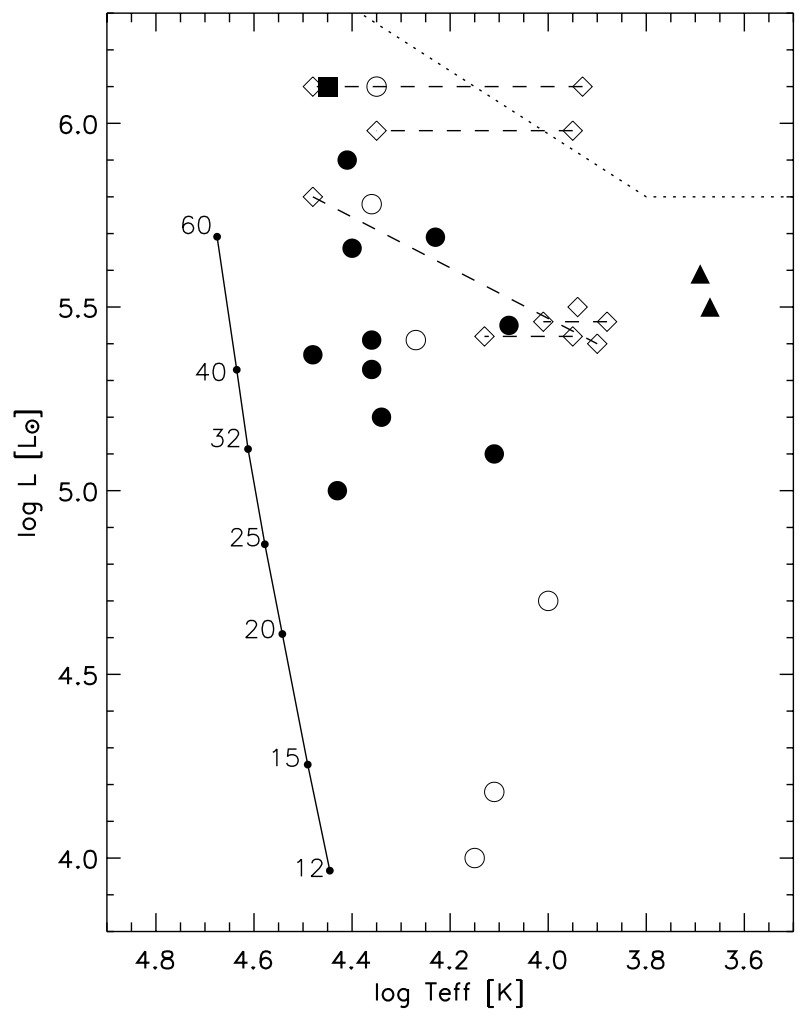

Fig. 8. Hertzsprung-Russell diagram (HRD) showing the location of each of our target stars. The start of the main sequence is indicated by the solid line, with specific initial masses from the evolutionary models of Ekström et al. (2012). Filled (open) symbols are stars with definite (absent or uncertain) CO band head emission. Based on the object types in Table 1, circles are B[e]SGs, diamonds represent LBVs, triangles show YHGs, and the square is the Peculiar Oe star. Dashed lines between symbols indicate LBV variable parameters. Also plotted is the Humphreys-Davidson limit (dotted line, Humphreys \& Davidson 1994).

line constitution of this group of stars is very different from the previous group, with some diversity among the members. Their appearance indicates cooler spectral types (late B and later). The strong Na I doublet emission in WRAY 15-751 and LHA 120S 116, combined with He I absorption and weak Mg II emission suggests a later B spectral type. These stars are currently in the so-called "active" state of their evolution. Outbursts have been reported recently for both WRAY 15-751 (Sterken et al. 2008) and LHA 120-S 155 (Gamen et al. 2009), confirming their active status. Further, the remarkable brightening we observe with respect to the original 2MASS magnitude measurements (see Sect. 2.3) reinforces this conclusion. Both LHA 115-S 52 (S 52) and LHA 120-S 116 lack sufficiently recent observations to confirm or deny this status, however the observed brightening of S 52 is suggestive. The study of Massey et al. (2007) for emission objects in M31 and M33 further corroborates these two spectral categories, labeling them "hot" and "cool" LBVs, based on their optical appearance.

Whether these low luminosity LBVs are currently in a postRSG phase is hard to tell, as they all appear to have similar initial masses of $\sim 40 M_{\odot}$, the boundary mass noted by Meynet et al. (2011), below which stars develop into LBVs as postRSGs. More massive stars become LBVs directly after the main sequence. 

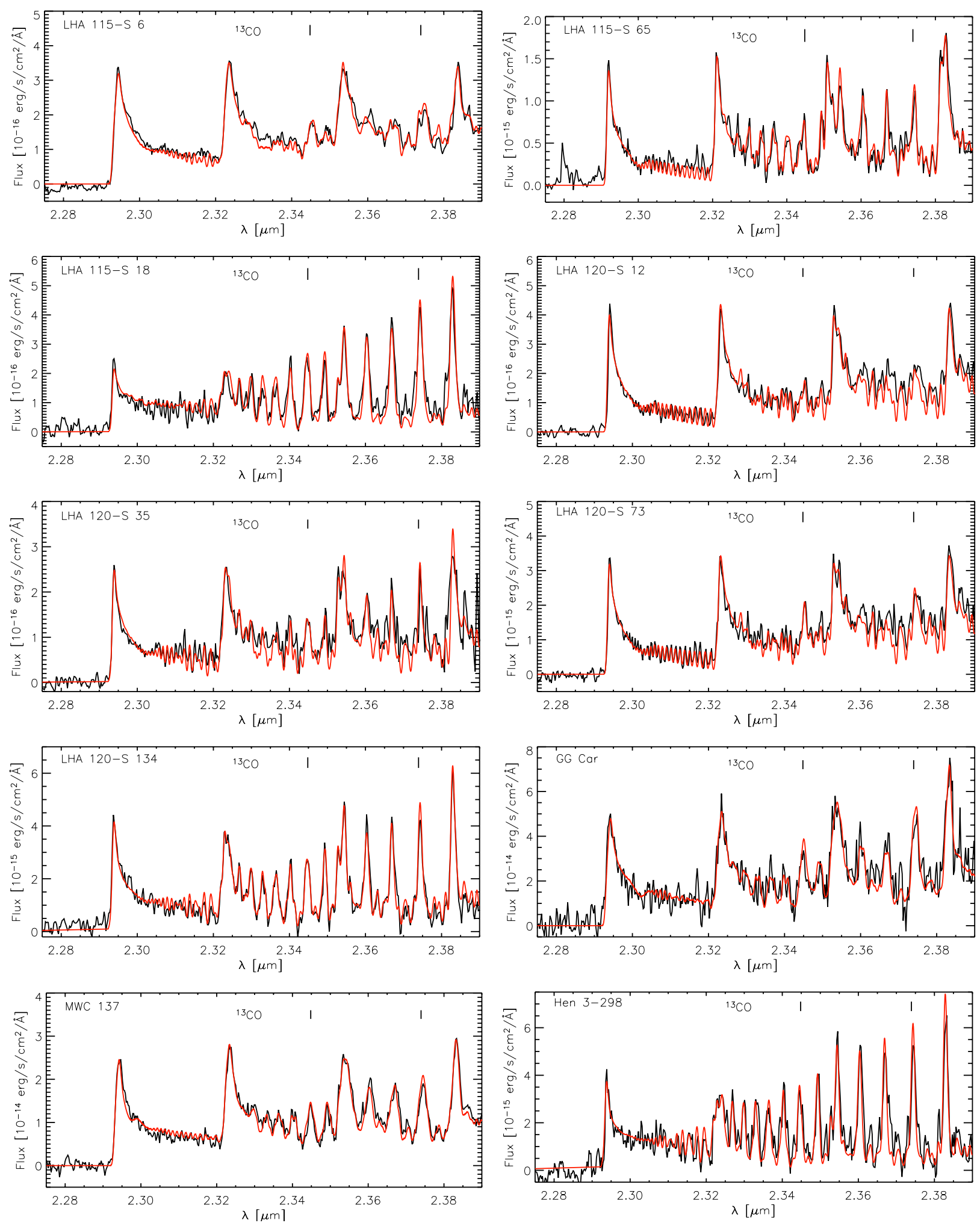

Fig. 9. Model fits (red) to the flux-calibrated, continuum subtracted CO band spectra (black) of B[e]SGs. The positions of the ${ }^{13} \mathrm{CO}$ band heads are indicated.

\subsection{2. $B[e] S G$ s and other objects}

As a group, the spectra of B[e]SGs fall into two categories based on spectral line composition. A majority of the objects display a hot spectrum, with strong helium and metal lines indicative of an early B spectral type. The remaining objects, LHA 120-S 73, LHA 120-S 35, LHA 120-S 59, and LHA 120-S 137, are likely later B supergiants, displaying fewer spectral lines, with some lines observed in absorption. These general assessments align with previous spectral type determinations from optical spectra as reported in Table 1.

One of the main results of the work presented in this paper is the comprehensive determination of the ${ }^{12} \mathrm{C} /{ }^{13} \mathrm{C}$ ratio for a variety of objects. While we cannot judge the evolutionary 

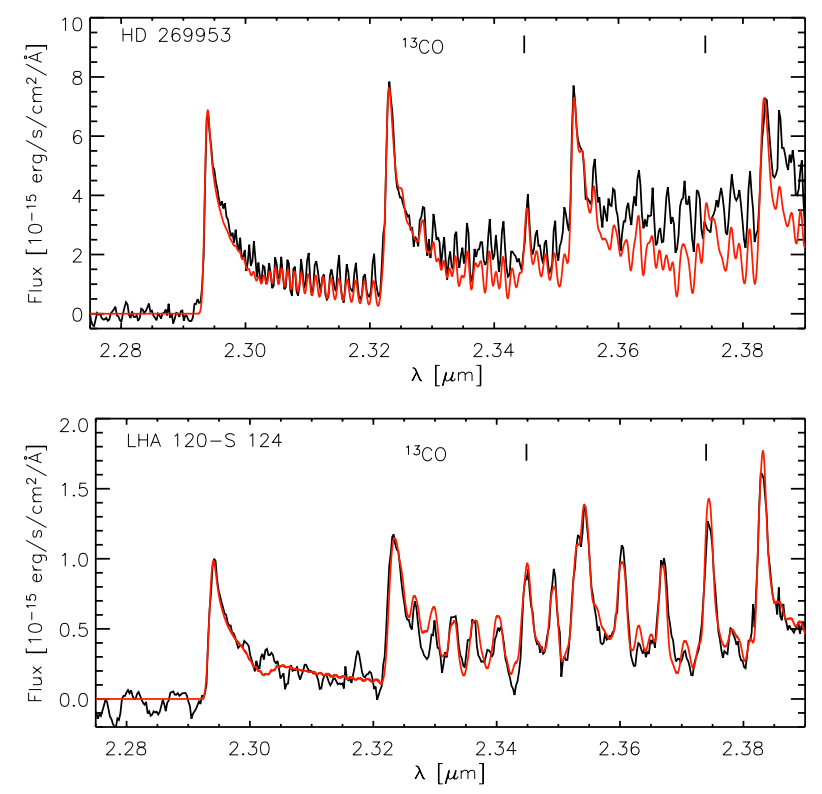

Fig. 10. Model fits (red) to the flux-calibrated, continuum subtracted CO band spectra (black) of the YHG HD 269953 and the Peculiar Oe star LHA $120-\mathrm{S} 124$. The positions of the ${ }^{13} \mathrm{CO}$ band heads are indicated.
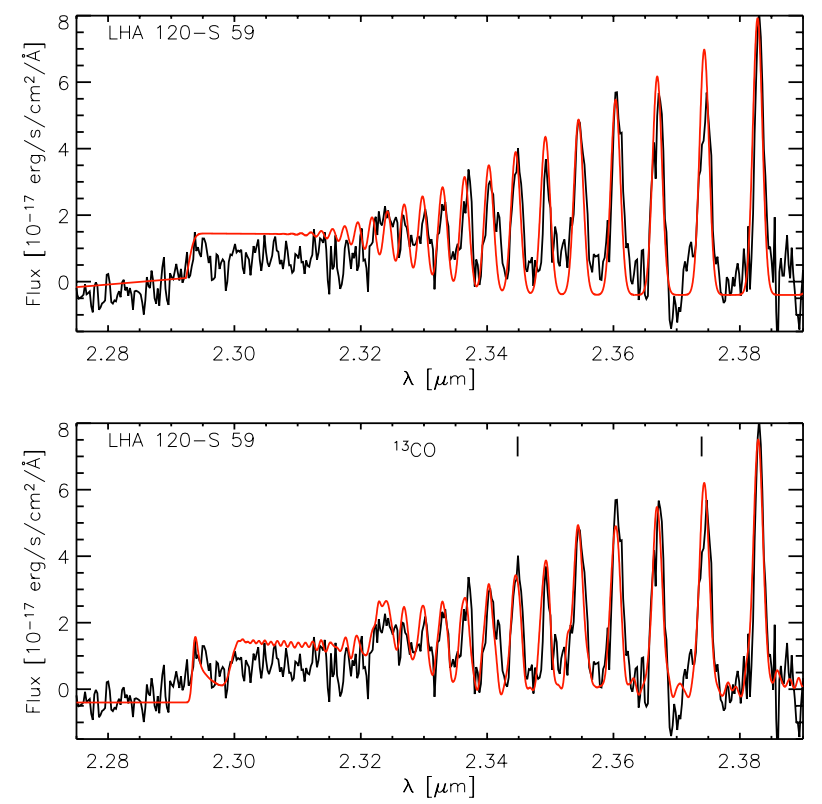

Fig. 11. Model fits (red) to the flux-calibrated, continuum subtracted CO band spectra (black) of the B[e]SG LHA 120-S 59. It is unclear whether a pure Pfund emission model (top) or a weak CO plus Pfund model (bottom) is the physically appropriate model. The positions of the ${ }^{13} \mathrm{CO}$ band heads are indicated.

status of $\mathrm{B}[\mathrm{e}] \mathrm{SG}$ that do not show any $\mathrm{CO}$ features, the presence and strength of the ${ }^{13} \mathrm{CO}$ molecular emission may be a tool to distinguish the ambiguity between objects that resemble both Herbig Ae/Be stars and B[e]SGs (shown previously by Liermann et al. 2010; Muratore et al. 2010).

The model derived ratios listed in Table 6, combined with the evolutionary tracks from Meynet \& Maeder (2005) and Ekström et al. (2012) shown in Fig. 12, provide evidence for the evolutionary status of each of these objects. Note that these tracks are for rotating models, as described in the figure caption. Identical tracks for non-rotating models show that the ${ }^{12} \mathrm{C} /{ }^{13} \mathrm{C}$ ratio does not drop below 20 until after the RSG phase, and reaches values $\leq 5$ for those tracks returning to the blue side of the HRD. Given the range of values observed in our objects, the nonrotating scenario does not seem to apply. The sample targets must therefore be rotating to achieve the surface chemical enrichment observed.

Although there are not as many tracks for the LMC and SMC objects, we can still get a preliminary idea of their evolutionary location based on the ${ }^{12} \mathrm{C} /{ }^{13} \mathrm{C}$ ratio. The majority of $\mathrm{B}$ [e]SGs appear, based on their ${ }^{13} \mathrm{C}$ enrichment, to be pre-RSG phase objects. However, the three stars with the lowest ${ }^{12} \mathrm{C} /{ }^{13} \mathrm{C}$ ratios, LHA 115-S 6, LHA 120-S 35, and LHA 120-S 73, may be close to entering the RSG phase or perhaps evolving blueward after their RSG phase. This distinction is difficult to ascertain without more information on the rotational speed of the star and/or without more evolutionary information for SMC and LMC metallicity stars. LHA $115-\mathrm{S} 6$ is a distinct case among these $\mathrm{B}[\mathrm{e}] \mathrm{SG}$ that has been suggested to be the result of a binary merger.

As expected, the YHG HD 269953 is confirmed to be a post-RSG object. Although we are unable to attain an accurate value for HD 269723, we may assume that it too is in a postRSG phase. The LMC Peculiar Oe star LHA 120-S 124 (S 124), which has also been suggested to be an LBV (van Genderen et al. 1998) and a peculiar O supergiant (Stahl \& Wolf 1987), can be presumed to have left the main-sequence, but not evolved past the RSG phase. S 124 could be considered an LBV candidate, due to its photometric variability, however, its $K$-band spectrum, and by extension its circumstellar environment, is much different than that of "bona fide" LBVs. Based on its stable CO emission, previously detected by McGregor et al. (1988b), the star more similarly resembles a B[e]SG, and could be considered a slightly hotter counterpart to these objects.

\subsection{Incidence of $\mathrm{CO}$ emission in B[e]SGs}

As a group, the $\mathrm{B}[\mathrm{e}]$ stars present similar spectral features, but with varying physical parameters and evolutionary stages (see e.g. Lamers et al. 1998). As an example, the B[e]SGs seem to be quite heterogeneous in the mass-loss traced by their circumstellar environments, with presumably the same mechanism forming their disk-like structure. While they may differ in mass and temperature, each object shows the same hybrid spectrum, forbidden emission, and infrared excess due to outer dust regions (e.g., Zickgraf et al. 1986; Bonanos et al. 2009, 2010; Kastner et al. 2010). One would thus expect that the $K$-band spectra, which contains information on the conditions of the circumstellar material, would be quite similar for each of these objects, and in the presence of dust, the disk structure would also form molecules. The result of this work that not all $\mathrm{B}[\mathrm{e}] \mathrm{SG}$ show $\mathrm{CO}$ emission is therefore surprising.

As evidenced by the HRD displayed in Fig. 8, there seems to be a lower luminosity limit as previously discussed. This may be due to lower mass-loss rates for these stars, leading to circumstellar environments which are of too low density to permit the detection of molecular emission. But, this limit does not explain the absence of $\mathrm{CO}$ emission in the higher luminosity stars LHA 120-S 127, LHA 120-S 89, and LHA 120-S 22. These stars appear in the HRD nearby to stars which show evidence for $\mathrm{CO}$ molecules. In the color-color diagram shown in Fig. 13, each non-CO emitting star is coincident with a $\mathrm{B}[\mathrm{e}] \mathrm{SG}$ with CO. B[e]SGs in the Galaxy, in the LMC, and in the SMC all show $\mathrm{CO}$ emission, eliminating metallicity as a determining factor. Our results may seem to suggest that only a portion of 


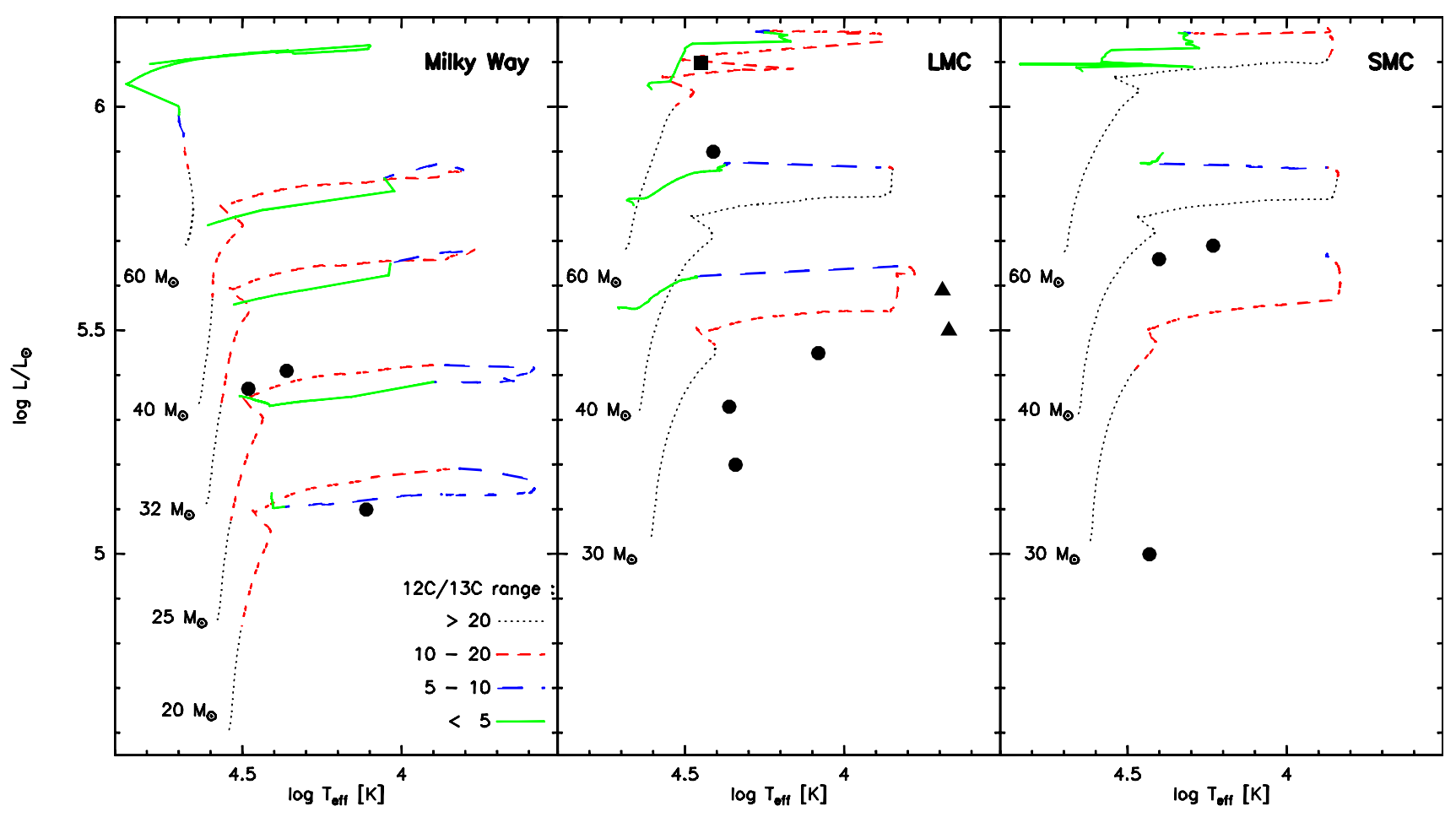

Fig. 12. Hertzsprung-Russell diagram showing the location of each target star with detected CO emission. The evolutionary tracks from Meynet \& Maeder (2005) (LMC, SMC) and Ekström et al. (2012) (Milky Way) trace the change in ${ }^{12} \mathrm{C} /{ }^{13} \mathrm{C}$ ratio with stellar evolution. For the Galaxy, the tracks are computed for an $\Omega=0.4$, while the Magellanic Cloud tracks are for $v_{\mathrm{eq}}=300 \mathrm{~km} \mathrm{~s}^{-1}$. $\Omega$ is the ratio of the rotational velocity to the critical rotation rate and $v_{\mathrm{eq}}$ is the equatorial rotational velocity. The symbols are explained in the caption of Fig. 8.

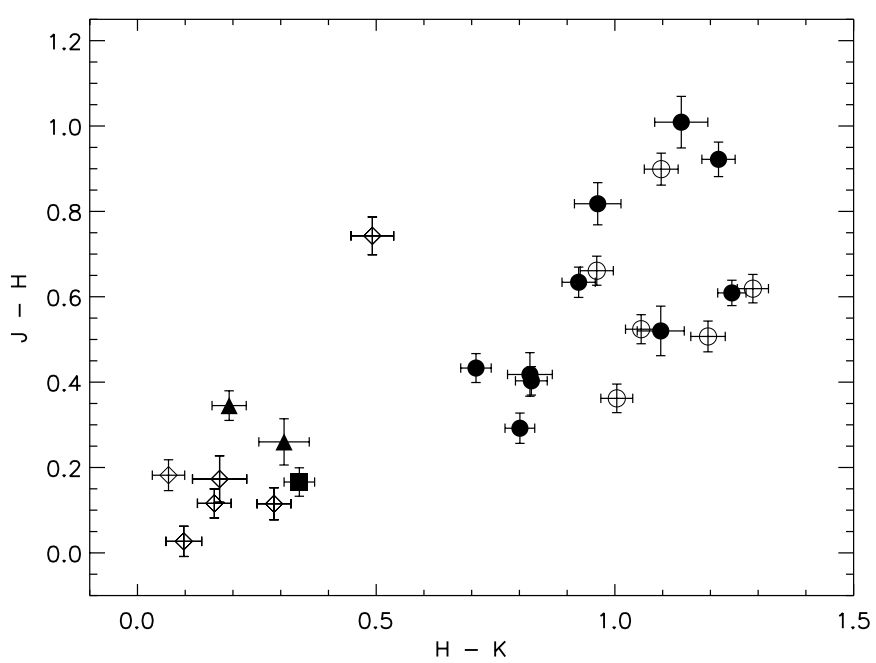

Fig. 13. Color-color diagram plotting each target's $J-H$ value and $H-K$ value calculated from the corresponding $J H K$ magnitudes listed in the 2MASS point source catalog (Cutri et al. 2003). The symbols are explained in the caption of Fig. 8. The B[e]SGs are all located on the right side of the figure, indicating the presence of dust. The other objects do not show such a large excess, with the exception of WRAY 15-751, which stands outside both groups due to a cool companion.

stars in the LMC do not show CO, but this should be seen as a small statistics issue, a problem only solved with the discovery of more group members.

We can speculate on the conditions which may be different in each of the stars in the sample without $\mathrm{CO}$ emission. One possibility is the structure of the circumstellar material may be different. Although $\mathrm{B}[\mathrm{e}] \mathrm{SG}$ s are presumed to possess dense disks, excellent locations for molecular formation, the current state of polarization studies only indicate that in a majority of these stars the material is not spherically distributed (Magalhães 1992; Oudmaijer \& Drew 1999; Melgarejo et al. 2001; Magalhães et al. 2006). Aret et al. (2012) find, based on $\mathrm{Ca}$ and $\mathrm{O}$ emission lines formed in the inner region of the circumstellar region, a spiral structure of material for LHA 120-S 22. The absence of hot $\mathrm{CO}$ emission could be related to the conditions of the material in the region where these molecules are formed, which may be at too low of a temperature or too low of a density for emission features to be detected in the spectrum. To investigate this, it would be prudent to obtain observations in different wavelength ranges to study the molecular content of the disks.

Another conceivable explanation is that these stars are not $\mathrm{B}[\mathrm{e}] \mathrm{SGs}$, but instead may be stars experiencing LBV conditions. For example, LHA 120-S 127 (S 127) is the brightest B[e]SG in our survey, and lies in the HRD very near to the HumphreysDavidson limit (Humphreys \& Davidson 1994), similar in position to the known LBVs LHA 120-S 128 and S Dor. As S 127 is viewed pole-on with respect to its circumstellar material (e.g., Aret et al. 2012), observations should reveal the entirety of the material composition. While studies show that S 127 has strong $\mathrm{Ca}$ and $\mathrm{O}$ emission in the inner region of its circumstellar environment (Aret et al. 2012) and copious amounts of dust further out (Bonanos et al. 2009; Kastner et al. 2010), no trace of $\mathrm{CO}$ emission is detected in our survey. As $\mathrm{CO}$ molecules form at a location intermediate to the $\mathrm{Ca}$ and $\mathrm{O}$ emitting material and the dust particles, the obvious conclusion is that the material surrounding S 127 is not a continuous disk, but a series of rings or shells. This type of structure is contrary to the hybrid wind model for B[e] stars and suggests episodic mass-loss events as in the case of $\mathrm{S}$ Dor ejections.

The role of binarity in the formation of disks and rings confined to the equatorial plane of evolved massive stars remains 
unclear. In the case of GG Car, its binary companion is close enough to interact, and the $\mathrm{CO}$ molecules are located in a circumbinary ring around the system. The origin of the material is likely from a main sequence remnant Be disk, but there is a small possibility of it being due to Roche lobe overflow (RLOF) due to the two stars close orbit (Marchiano et al. 2012; Kraus et al. 2013). The Galactic B[e]SG HD 327083 (Wheelwright et al. 2012) is another example of a system with circumbinary material similar to GG Car, as well as detected CO emission. In these two cases, the stable presence of the $\mathrm{CO}$ emission and the appearance of the material in a detached ring, may be a consequence of the binary interaction. As this study finds detached circumstellar structure, evidenced by cooler $\mathrm{CO}$ emitting gas, it would be straightforward to make the conclusion that all stars with $\mathrm{CO}$ emission exist in close binary systems. However, there are several counter points to disparage binarity as a factor in the formation of disks or rings. The star MWC 137 has no visible companion (Marston \& McCollum 2008), and yet shows detectable ${ }^{12} \mathrm{CO}$ and ${ }^{13} \mathrm{CO}$. LHA 115 -S 18 was suggested to have a binary companion, but no observational evidence backs up such a claim (Torres et al. 2012). Similarly, CPD-52 9243 (Cidale et al. 2012) has been suggested to be a binary, but there has been no confirmation. LHA 115-S 6 has a binary companion, however, the orbital distance is large and there is no possibility of RLOF interaction between the two objects (Zickgraf et al. 1996). No binarity has been indicated for any of the other COemitting stars, and thus at this point we are hesitant to place any confidence in the role of binarity as a circumstellar disk formation and maintenance mechanism. More work in this aspect of $\mathrm{B}[\mathrm{e}]$ supergiant research may prove incredibly insightful with the increasing capabilities of interferometry.

\subsection{Influence of rotation on stellar mass loss}

The detection of Keplerian rotating disk material around some B[e]SGs (Kraus et al. 2010; Wheelwright et al. 2012; Cidale et al. 2012) is in direct opposition to the outflowing disk expected by the model of Zickgraf et al. (1985). Further, the general stability of these disks, and in particular the presence of dust particles, indicate that the material is not in constant motion, as in an outflowing disk. While this initial model may not be physically applicable, rotation, more specifically rapid-rotation, may play a large role in the details of the stellar mass loss.

Viscous disks have been studied in the context of classical Be stars and suggested for the case of S 65 (Oksala et al. 2012). This star is particularly interesting since the CO emission appears to be traveling at a higher outward speed than other parts of the circumstellar environment. The $\mathrm{CO}$ band heads required an additional shift in velocity of $196 \mathrm{~km} \mathrm{~s}^{-1}$ to simultaneously fit the CO band head emission and the Pfund emission. This star is a confirmed rapid rotator (Kraus et al. 2010), rotating at a minimum of $75 \%$ of the critical speed. Could the rapid stellar rotation be the cause of this high velocity ejection? S 65 is thought to be viewed disk edge on, and thus it could be rotating at a nearly critical velocity, considering the $v \sin i$ measurement was obtained from photospheric line profiles, which are unable to determine rotation speeds higher than $75 \%$ of critical (Townsend et al. 2004). If we consider that the star may be critically rotating, it could have ejected a large amount of mass via the process described by Kurfürst \& Krtička (2012), with the star subsequently spinning down due to the large loss of angular momentum. While this situation has potential, the only way to determine the physical explanation is new observations. The possibility also still exists that this object is an LBV and we are viewing the result of an outburst.

LHA 120-S 73 is another star with a determined rotation velocity $\left(v \sin i \sim 50 \mathrm{~km} \mathrm{~s}^{-1}\right.$ ). Aret et al. (2012) found the star may be oriented more or less pole-on with $i=28^{\circ}$. This would indicate a rotation at $\sim 75 \%$ critical, nearly identical to $S 65$. Besides the cause of the mass-loss, another possible effect of rapid rotation in these stars is the composition of the ejected material. As the rotation rate increases, the chemical enrichment achieved during evolution is mixed and transported to the stellar surface at a higher rate. Two similarly evolved stars, one with a much higher rotation rate, may have very different ${ }^{12} \mathrm{C} /{ }^{13} \mathrm{C}$ ratios. This could be the explanation of the higher ratios found in S 73 and S 35 (although the $v$ sin $i$ of this star has not been determined). An alternative explanation presumes that the $\mathrm{B}[\mathrm{e}] \mathrm{SG}$ are all in the post-RSG phase, with the stars with lower amounts of ${ }^{13} \mathrm{CO}$ initially slowly or non-rotating. This scenario is much less likely considering the blueward location of these objects in the HRD, and the known rotational velocity of S 65 .

\subsection{Evolutionary connection between transition objects}

The connection of these transition objects within their evolutionary progression was one of the initial primary objectives of this study, as well as previous studies by McGregor et al. (1988a,b, 1989) and Morris et al. (1996). LHA 115-S 18 (S 18), a supergiant in the SMC, has been the prime example of the possible link between the seemingly stable B[e]SGs and the variable LBV stars. Variability of the star has been found in a number of different observations. The supergiant S 124 similarly shows many characteristics that may indicate it is in an LBV state, however, the stable presence of $\mathrm{CO}$ emission leaves some ambiguity. In an exceptional case, Morris et al. (1997) found variable CO emission in the $K$-band spectrum of the known LBV HR Car, giving further proof of the hazy distinction between $\mathrm{B}[\mathrm{e}] \mathrm{SGs}$ and LBVs. The presence of CO emission in LBVs may be observable only at very specific short instances in time, based on the speed and physical conditions of the ejected material. In this vein, we suggest that the cause of the detached material may be a outburst, or ejection of material from the star, possibly in an SDor type event, or due to some other physical mechanism such as pulsations.

As many of the classifications of LBV candidates and $\mathrm{B}[\mathrm{e}] \mathrm{SGs}$ are based on few observations, there can be confusion when there are spectral similarities. For example, the B[e]SG S 65, showed no variability in optical spectra, however, recent work by Oksala et al. (2012) find that CO molecular emission appeared suddenly in the $K$-band spectrum of this star within a period of 9 months. This rapid development of spectral features indicates changing conditions in the circumstellar environment. The short time frame of its appearance combined with the large observed velocity of the material suggests an outburst of material, reminiscent of an S-Dor-type event. Further, Kraus et al. (2010) determined that this object is located close to the LBV minimum instability strip (Groh et al. 2009). But, this is not the only example of possible LBV behavior seen in B[e]SGs. The spiral arm structure seen in optical spectra of LHA 120-S 22 by Aret et al. (2012), as well as the series of ring material detected around S 127, point to multiple mass ejections suggestive of a series of S-Dor-type ejections. Variability exists in several $\mathrm{B}$ [e]SGs, however the study of this group of objects suffers from lack of data, with few follow up observations.

Morris et al. (1996) inferred that the distinction between $\mathrm{B}$ [e]SGs and other transition objects is a higher rotational 
velocity, resulting in a more intensely non-spherical wind, and thus disk, a theory supported by the work of Schulte-Ladbeck et al. (1993). However, a recent study by Groh et al. (2009) determined that LBVs such as HR Car and AG Car are in fact rotating at a significant percentage of their critical velocities. It is possible that LBVs and B[e]SGs are the same type of stars, with some $\mathrm{B}[\mathrm{e}] \mathrm{SG}$ not having yet experienced any LBV-type outbursts.

One glaring difference between the group of LBVs and $\mathrm{B}$ [e]SGs is the extreme IR excess seen in the color-color diagram (see Fig. 13). This IR excess indicates that B[e]SGs are surrounded by large amounts of hot dust, whereas the LBVs only host marginal amounts of hot dust, but huge amounts of cool dust at far distances. This figure shows 2MASS measurements, and is not necessary representative of the values at the time of observations. Since we do not have access to the $J$ and $H$-band magnitudes at that epoch, we cannot postulate on any differences, but it is likely that these objects will not have a substantial change in position. Besides the decreased amount of hot dust and the lack of $\mathrm{CO}$ band head emission, the $K$-band spectra of quiescent LBVs are otherwise indistinguishable from the B[e]SGs (see Fig. 7).

\section{Conclusions}

In this paper, a survey of $K$-band spectra from the SINFONI spectrograph has been presented. Our 25 survey targets include LBVs, B[e]SGs, YHGs, and a Peculiar Oe star from the Galaxy, the LMC, and the SMC. In the following, we summarize the main results of this study, as well as make suggestions for future studies.

- The $K$-band spectra of LBVs in their quiescent or "hot" phase are similar in appearance to B[e]SGs. LBVs in their active or "cool" phase have $K$-band spectra identical to those observed for YHGs. These similarities include both continuum shape and spectral composition, with the exception of $\mathrm{CO}$ emission, not found in the spectrum of any of our LBV samples.

- CO band head emission was detected in 13 of our targets, 10 of which are B[e]SGs, 2 YHGS, and 1 Peculiar Oe star. For 3 of these stars (MWC 137, LHA 120-S 35, and LHA 115S 65), this survey presents the initial discovery of this feature (the appearance of CO in S 65 has been presented in Oksala et al. 2012). There appears to be a lower luminosity limit, $\log L / L_{\odot}=5.0$, below which no CO emission is detected.

- Model fits to the spectrum reveal the temperature and density of the $\mathrm{CO}$ emitting region. For each object, we find that the $\mathrm{CO}$ emitting region must be located in a detached disk or ring structure, rather than in a continuous, outflowing disk extending from the stellar surface out to far distances.

- Each object with visible ${ }^{12} \mathrm{CO}$ band head emission also shows ${ }^{13} \mathrm{CO}$ emission, indicating evolution past the end of the main sequence. Based on the enrichment of ${ }^{13} \mathrm{C}$, a majority of the stars appear to be in a pre-RSG phase, evolving redward across the HRD. YHGs are post-RSG objects preparing to evolve blueward, assuming that stars are able to pass through the Yellow Void. B[e]SGs are therefore not the descendants of YHGS.

- Several of the targets regarded as B[e]SGs appear to be indistinguishable from LBVs, and may be regarded as LBV candidates in quiescence. Variability in several B[e]SGs also suggest that they may be candidates as well, further blurring the distinction between these two ambiguous groups.
This survey highlights certain areas where future work can concentrate for these enigmatic groups of stars. High resolution spectra of CO band heads can reveal the kinematics of the disk structure where these molecules are located. Simultaneous disk diagnostics, such as [Ca II] and [O I] lines and the $\mathrm{CO}$ band head emission, are necessary to globally examine the kinematic, temperature, and density structure of the circumstellar environments of evolved massive stars. To determine the stability of the circumstellar material of these stars over time and determine the nature of each object (LBV, B[e]SG, YHG, etc.), further monitoring of the $K$-band spectra (and other spectral ranges) of all of these objects is necessary.

Acknowledgements. The authors would like to express their gratitute to Dr. Adriane Liermann for her assistance in computing the $K_{\mathrm{s}}$-band magnitudes. This research made use of the NASA Astrophysics Data System (ADS). M.E.O. and M.K. acknowledge financial support from GAČR under grant number 209/11/1198. The Astronomical Institute Ondřejov is supported by the project RVO:67985815. M.B.F. acknowledges Conselho Nacional de Desenvolvimento Científico e Tecnológico (CNPq-Brazil) and Ministério de Ciência, Tecnologia e Inovação (MCTI) for a PCI-D grant. L.C. acknowledges financial support from the Agencia de Promoción Científica y Tecnológica (Préstamo BID PICT 2011/0885), PIP 0300 CONICET, and the Programa de Incentivos G11/109 of the Universidad Nacional de La Plata, Argentina. Financial support from International Cooperation of the Czech Republic (MŠMT, 7AMB12AR021) and Argentina (Mincyt-Meys, ARC/11/10) is acknowledged. M.F.M. is a research fellow of the Universidad Nacional de La Plata, Argentina.

\section{References}

Aret, A., Kraus, M., Muratore, M. F., \& Borges Fernandes, M. 2012, MNRAS, 423, 284

Bonanos, A. Z., Massa, D. L., Sewilo, M., et al. 2009, AJ, 138, 1003

Bonanos, A. Z., Lennon, D. J., Köhlinger, F., et al. 2010, AJ, 140, 416

Bonnet, H., Abuter, R., Baker, A., et al. 2004, The Messenger, 117, 17

Cidale, L. S., Borges Fernandes, M., Andruchow, I., et al. 2012, A\&A, 548, A72

Clark, J. S., Arkharov, A., Larionov, V., et al. 2011, BSRSL, 80, 361

Curé, M. 2004, ApJ, 614, 929

Curé, M., \& Rial, D. F. 2004, A\&A, 428, 545

Curé, M., Rial, D. F., \& Cidale, L. 2005, A\&A, 437, 929

Cutri, R. M., Skrutskie, M. F., van Dyk, S., et al. 2003, VizieR Online Data Catalog, II/246

de Jager, C. 1998, A\&ARv, 8, 145

Eisenhauer, F., Abuter, R., Bickert, K., et al. 2003, in Instrument Design and Performance for Optical Infrared Ground-based Telescopes, eds. M. Iye, \& A. F. M. Moorwood, SPIE, 4841, 1548

Ekström, S., Georgy, C., Eggenberger, P., et al. 2012, A\&A, 537, A146

Esteban, C., \& Fernández, M. 1998, MNRAS, 298, 185

Gamen, R., Barba, R., Walborn, N., et al. 2009, IAUC, 9082, 1

Groh, J. H., Damineli, A., Hillier, D. J., et al. 2009, ApJ, 705, L25

Gummersbach, C. A., Zickgraf, F.-J., \& Wolf, B. 1995, A\&A, 302, 409

Hillenbrand, L. A., Strom, S. E., Vrba, F. J., \& Keene, J. 1992, ApJ, 397, 613

Howarth, I. D. 1983, MNRAS, 203, 301

Hu, J. Y., de Winter, D., The, P. S., \& Perez, M. R. 1990, A\&A, 227, L17

Humphreys, R. M., \& Davidson, K. 1994, PASP, 106, 1025

Kastner, J. H., Buchanan, C., Sahai, R., Forrest, W. J., \& Sargent, B. A. 2010, AJ, 139, 1993

Kraus, M. 2006, A\&A, 456, 151

Kraus, M. 2009, A\&A, 494, 253

Kraus, M., \& Lamers, H. J. G. L. M. 2003, A\&A, 405, 165

Kraus, M., Krügel, E., Thum, C., \& Geballe, T. R. 2000, A\&A, 362, 158

Kraus, M., Borges Fernandes, M., Kubát, J., \& Araújo, F. X. 2008, A\&A, 487, 697

Kraus, M., Borges Fernandes, M., \& Araújo, F. X. 2010, A\&A, 517, A30

Kraus, M., Oksala, M. E., Nickeler, D. H., et al. 2013, A\&A, 549, A28

Kurfürst, P., \& Krtička, J. 2012, in Circumstellar Dynamics at High Resolution, eds. A. C. Carciofi, \& Th. Rivinius, ASP Conf. Ser., 464

Kurucz, R. L. 1993, VizieR Online Data Catalog, VI/039

Lamers, H. J. G, \& Pauldrach, A. W. A. 1991, A\&A, 422, L5

Lamers, H. J. G. L. M., Zickgraf, F.-J., de Winter, D., Houziaux, L., \& Zorec, J. 1998, A\&A, 340, 117

Leitherer, C., Appenzeller, I., Klare, G., et al. 1985, A\&A, 153, 168

Liermann, A., Hamann, W.-R., \& Oskinova, L. M. 2009, A\&A, 494, 1137 
M. E. Oksala et al.: Probing the ejecta of evolved massive stars in transition

Liermann, A., Kraus, M., Schnurr, O., \& Borges Fernandes, M. 2010, MNRAS, 408, L6

Maeder, A., \& Meynet, G. 2004, IAUS, 215, 500

Magalhães, A. M. 1992, ApJ, 398, 286

Magalhães, A. M., Melgarejo, R., Pereyra, A., \& Carciofi, A. C. 2006, in Stars with the $\mathrm{B}[\mathrm{e}]$ Phenomenon, eds. M. Kraus, \& A. S. Miroshnichenko, ASP Conf. Ser., 355, 147

Marchiano, P., Brandi, E., Muratore, M. F., et al. 2012, A\&A, 540, A91

Marston, A. P., \& McCollum, B. 2008, A\&A, 477, 193

Massey, P., McNeill, R. T., Olsen, K. A. G., et al. 2007, AJ, 134, 2474

McGregor, P. J., Hillier, D. J., \& Hyland, A. R. 1988a, ApJ, 334, 639

McGregor, P. J., Hyland, A. R., \& Hillier, D. J. 1988b, ApJ, 324, 1071

McGregor, P. J., Hyland, A. R., \& McGinn, M. T. 1989, A\&A, 223, 237

Melgarejo, R., Magalhães, A. M., Carciofi, A. C., \& Rodrigues, C. V. 2001, A\&A, 377, 581

Meynet, G., \& Maeder, A. 2005, A\&A, 429, 581

Meynet, G., Georgy, C., Hirschi, R., et al. 2011, BSRSL, 80, 266

Miroshnichenko, A. S., Bjorkman, K. S., Grosso, M., et al. 2005, A\&A, 436, 653

Morris, P. W., Eenens, P. R. J., Hanson, M. M., Conti, P. S., \& Blum, R. D. 1996, ApJ, 470, 597

Morris, P. W., Voors, R. H. M, Lamers, H. J. G. L. M., \& Eenens, P. R. J. 1997, in Luminous Blue Variables: Massive Stars in Transition Astron. Soc. Pac., eds. A. Nota, H. J. G. L. M. Lamers, ASP Conf. Ser., 120, 20

Muratore, M. F., Kraus, M., Liermann, A., et al. 2010, BAAA, 53, 123

Muratore, M. F., Kraus, M., Oksala, M. E., et al. 2013, A\&A, submitted

Nota, A., \& Lamers, H., 1997, ASP Conf. Ser., 120
Oksala, M. E., Kraus, M., Arias, M. L., et al. 2012, MNRAS, 426, L56

Oudmaijer, R. D., \& Drew, J. E. 1999, MNRAS, 305, 166

Pelupessy, I., Lamers, H. J. G. L. M., \& Vink, J. S. 2000, A\&A, 359, 695

Schulte-Ladbeck, R. E., Leitherer, C., Clayton, G. C., et al. 1993, ApJ, 407, 723

Skrutskie, M. F., Cutri, R. M., Stiening, R., et al. 2006, AJ, 131, 1163

Stahl, O., \& Wolf, B. 1987, A\&A, 181, 293

Sterken, C., van Genderen, A. M., Plummer, A., \& Jones, A. F. 2008, A\&A, 484, 463

Torres, A. F., Kraus, M., Cidale, L. S., et al. 2012, MNRAS, 427, L80

Townsend, R. H. D., Owocki, S. P., \& Howarth, I. D. 2004, MNRAS, 350, 189 van Genderen, A. M., Sterken, C., De Groot, M., \& Reijns, R. A. 1998, A\&A, 332,857

Wheelwright, H. E., de Wit, W. J., Weigelt, G., Oudmaijer, R. D., \& Ilee, J. D. 2012, A\&A, 543, A77

Zickgraf, F.-J. 2000, in The Be phenomenon in early-type stars, eds. M. A. Smith, H. F. Henrichs, \& J. Fabregat, ASP Conf. Ser., 214, 26

Zickgraf, F.-J. 2006, in Stars with the B[e] Phenomenon. eds. M. Kraus, \& A. S. Miroshnichenko, ASP Conf. Ser., 355, 135

Zickgraf, F.-J., \& Wolf, B. 1987, IAUS, 122, 439

Zickgraf, F.-J., Wolf, B., Stahl, O., Leitherer, C., \& Klare, G. 1985, A\&A, 143, 421

Zickgraf, F.-J., Wolf, B., Leitherer, C., Appenzeller, I., \& Stahl, O. 1986, A\&A, 163,119

Zickgraf, F.-J., Wolf, B., Stahl, O., \& Humphreys, R. M. 1989, A\&A, 220, 206

Zickgraf, F.-J., Kovacs, J., Wolf, B., et al. 1996, A\&A, 309, 505 

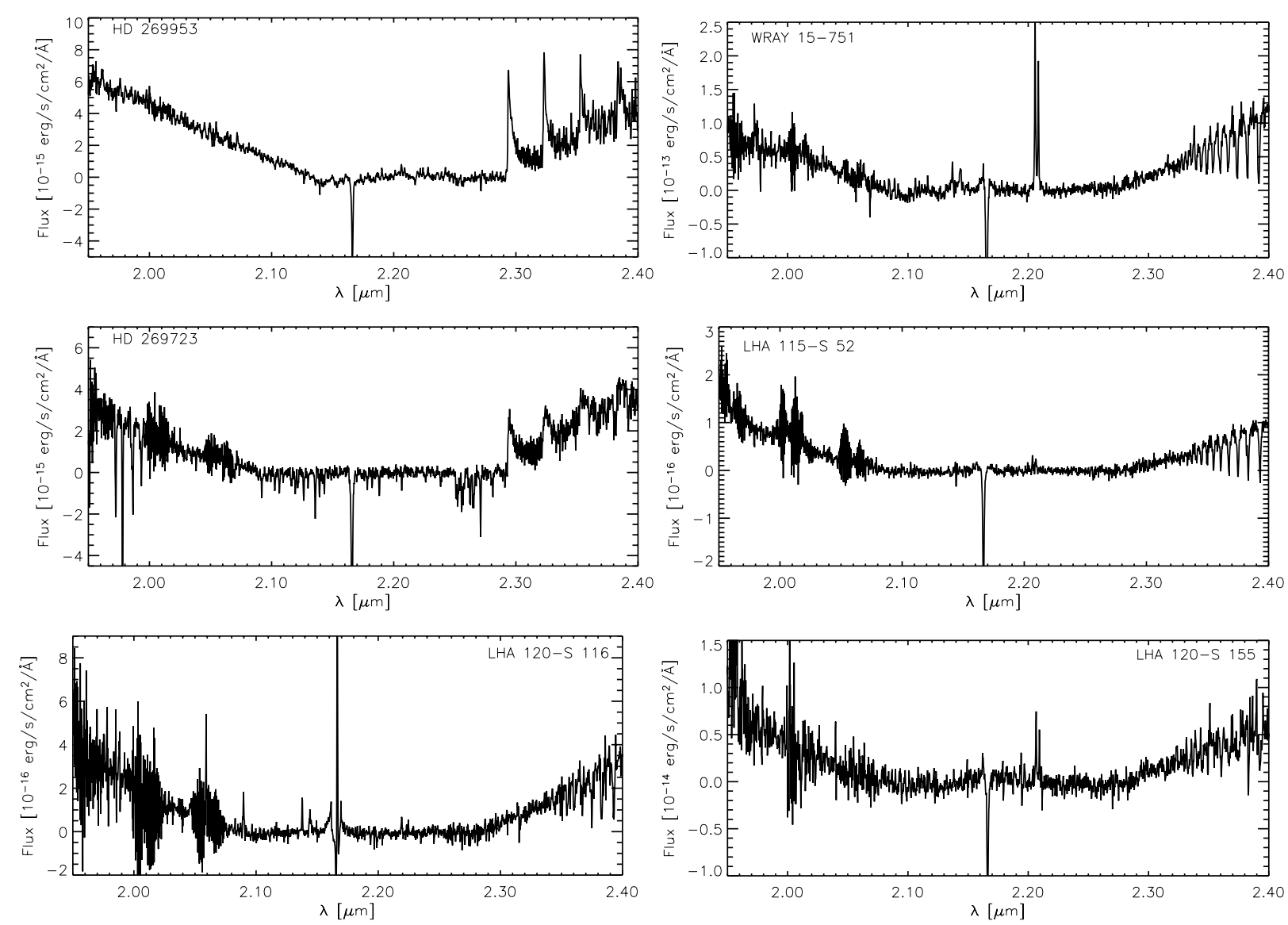

Fig. 6. Objects with cool supergiant type spectra. Flux-calibrated, continuum subtracted SINFONI $K$-band spectra of the YHGs HD 269953 and HD 269723 and the LBVs WRAY 15-751, LHA 115-S 52, LHA 120-S 116, LHA 120-S 155. 


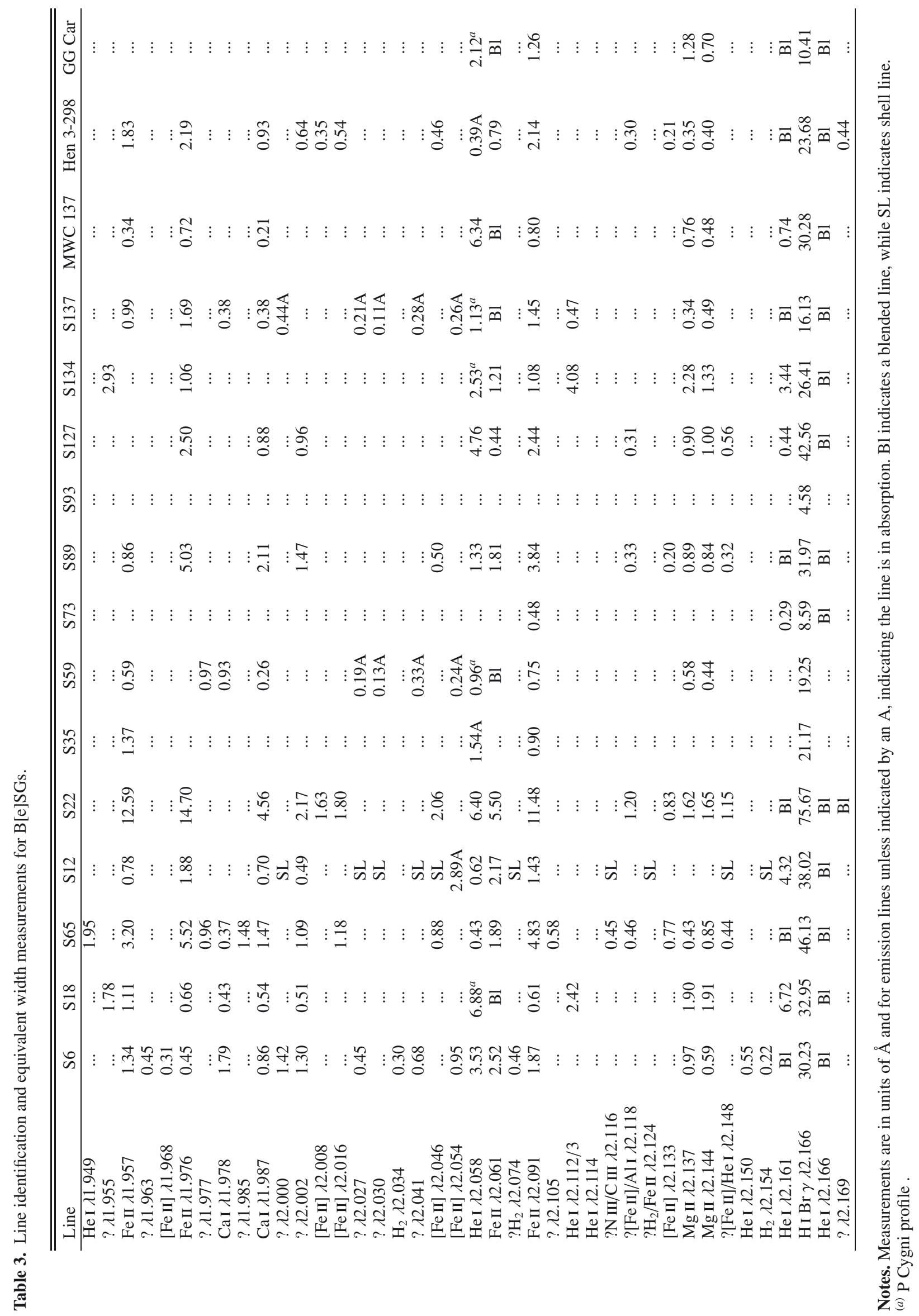




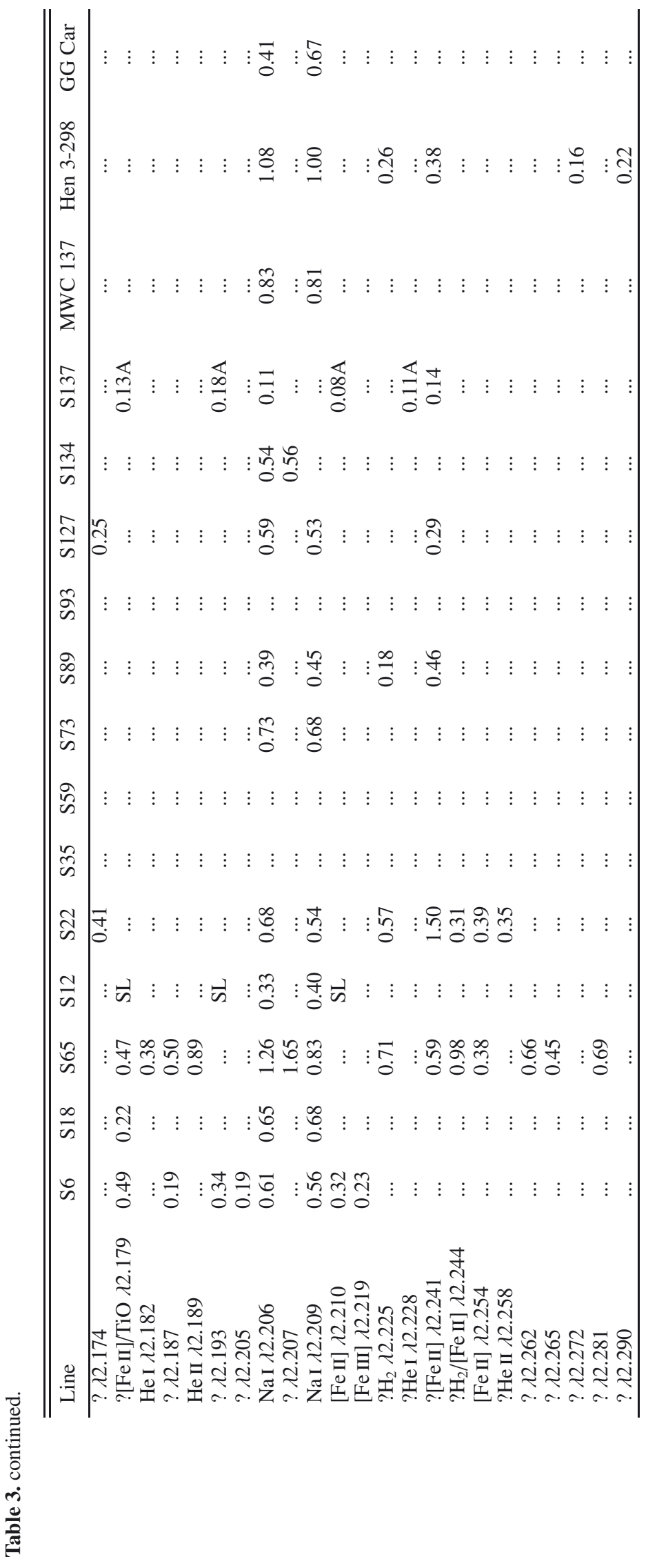


M. E. Oksala et al.: Probing the ejecta of evolved massive stars in transition

Table 4. Line identification and equivalent width measurements for YHGs and Peculiar Oe.

\begin{tabular}{lccc}
\hline \hline Line & 269723 & 269953 & S124 \\
\hline$? \lambda 1.955$ & $\ldots$ & $\ldots$ & 2.62 \\
$? \lambda 1.976$ & $1.10 \mathrm{~A}$ & $\ldots$ & 1.36 \\
Ca I $\lambda 1.978$ & $1.81 \mathrm{~A}$ & $\ldots$ & 0.83 \\
Ca I $\lambda 1.987$ & $1.27 \mathrm{~A}$ & $\ldots$ & 2.27 \\
$? \lambda 1.993$ & $0.85 \mathrm{~A}$ & $\ldots$ & 1.04 \\
He I $\lambda 2.058$ & $\ldots$ & $\ldots$ & 45.33 \\
Fe II $\lambda 2.091$ & $1.65 \mathrm{~A}$ & $\ldots$ & $\ldots$ \\
$? \lambda 2.103$ & $0.21 \mathrm{~A}$ & $\ldots$ & $\ldots$ \\
$? \lambda 2.107$ & $0.23 \mathrm{~A}$ & $\ldots$ & $\ldots$ \\
He I $\lambda 2.112 / 3$ & $\ldots$ & $\ldots$ & 3.78 \\
$? \lambda 2.120$ & $0.26 \mathrm{~A}$ & $\ldots$ & $\ldots$ \\
$? \lambda 2.127$ & $0.30 \mathrm{~A}$ & $\ldots$ & $\ldots$ \\
$? \lambda 2.130$ & $0.13 \mathrm{~A}$ & $\ldots$ & $\ldots$ \\
Mg II $\lambda 2.137$ & $0.53 \mathrm{~A}$ & $\ldots$ & 1.28 \\
$? \lambda 2.140$ & $0.29 \mathrm{~A}$ & $\ldots$ & $\ldots$ \\
Mg II $\lambda 2.144$ & $0.23 \mathrm{~A}$ & $\ldots$ & 0.70 \\
He I $\lambda 2.161$ & $\ldots$ & $\ldots$ & 3.01 \\
H I Br $\gamma \lambda 2.166$ & $3.69 \mathrm{~A}$ & $3.40 \mathrm{~A}$ & 21.46 \\
He I $\lambda 2.166$ & $\mathrm{Bl}$ & $\mathrm{Bl}$ & $\mathrm{Bl}$ \\
$?[\mathrm{Fe}$ II]/TiO $\lambda 2.179$ & $0.18 \mathrm{~A}$ & $\ldots$ & 0.47 \\
He I $\lambda 2.182$ & $0.22 \mathrm{~A}$ & $\ldots$ & $\ldots$ \\
$? ? \mathrm{He}$ II $\lambda 2.188$ & $0.35 \mathrm{~A}$ & $\ldots$ & $\ldots$ \\
Na I $\lambda 2.206$ & $0.22 \mathrm{~A}$ & $\ldots$ & 2.54 \\
Na I $\lambda 2.209$ & $\ldots$ & $\ldots$ & 2.47 \\
$? \lambda 2.251$ & $2.22 \mathrm{~A}$ & $\ldots$ & $\ldots$ \\
$? \lambda 2.253$ & $\mathrm{~B} 1$ & $\ldots$ & $\ldots$ \\
{$[$ Fe II] $\lambda 2.254$} & $\mathrm{B} 1$ & $\ldots$ & $\ldots$ \\
$? \lambda 2.256$ & $\mathrm{~B} 1$ & $\ldots$ & $\ldots$ \\
$? \lambda 2.257$ & $\mathrm{~B} 1$ & $\ldots$ & $\ldots$ \\
$?$ He II $\lambda 2.258$ & $\mathrm{~B} 1$ & $\ldots$ & $\ldots$ \\
$? \lambda 2.262$ & $0.37 \mathrm{~A}$ & $\ldots$ & $\ldots$ \\
$? \lambda 2.265$ & $0.67 \mathrm{~A}$ & $\ldots$ & $\ldots$ \\
$? \lambda 2.272$ & $0.77 \mathrm{~A}$ & $\ldots$ & $\ldots$ \\
\hline & & & \\
& &
\end{tabular}

Notes. Measurements are in units of $\AA$ and for emission lines unless indicated by an $\mathrm{A}$, indicating the line is in absorption. Bl indicates a blended line. 
A\&A 558, A17 (2013)

Table 5. Line identification and equivalent width measurements for LBVs.

\begin{tabular}{|c|c|c|c|c|c|c|}
\hline Line & $\overline{\text { S52 }}$ & S96 & S116 & S128 & S155 & $\overline{\text { WRAY751 }}$ \\
\hline Fe II $\lambda 1.958$ & $\ldots$ & 5.00 & $\ldots$ & 4.24 & $\ldots$ & $\ldots$ \\
\hline Fe II $\lambda 1.976$ & $\ldots$ & 14.84 & $\ldots$ & 9.46 & $\ldots$ & $\ldots$ \\
\hline Са I $\lambda 1.978$ & $\ldots$ & $\ldots$ & $\ldots$ & 0.53 & $\ldots$ & $\ldots$ \\
\hline$? \lambda 1.985$ & $\ldots$ & $\ldots$ & $\ldots$ & 0.44 & $\ldots$ & $\ldots$ \\
\hline Ca I $\lambda 1.987$ & $\ldots$ & 4.15 & $\ldots$ & 3.05 & $\ldots$ & $\ldots$ \\
\hline$? \mathrm{H}_{2} \lambda 2.002$ & $\ldots$ & 4.46 & $\cdots$ & 2.42 & ... & $\ldots$ \\
\hline [Fe II] $\lambda 2.008$ & $\ldots$ & 1.15 & $\ldots$ & 0.59 & $\ldots$ & $\ldots$ \\
\hline [Fe II] $\lambda 2.016$ & $\ldots$ & 1.09 & $\ldots$ & 0.91 & $\ldots$ & $\ldots$ \\
\hline$? \lambda 2.020$ & $\ldots$ & 0.69 & $\ldots$ & $\ldots$ & $\ldots$ & $\ldots$ \\
\hline$? \lambda 2.030$ & $\ldots$ & 0.43 & $\ldots$ & $\ldots$ & $\ldots$ & $\ldots$ \\
\hline $\mathrm{H}_{2} \lambda 2.034$ & $\ldots$ & 0.17 & $\ldots$ & $\ldots$ & $\ldots$ & $\ldots$ \\
\hline ?He II $\lambda 2.037$ & $\ldots$ & 0.48 & $\ldots$ & $\ldots$ & $\ldots$ & $\ldots$ \\
\hline [Fe II] $\lambda 2.046$ & $\ldots$ & 1.10 & $\ldots$ & 1.33 & $\ldots$ & $\ldots$ \\
\hline [Fe II] $\lambda 2.054$ & $\ldots$ & 1.11 & $\ldots$ & 0.72 & $\ldots$ & $\ldots$ \\
\hline He I $\lambda 2.058$ & $\ldots$ & 6.22 & 0.52 & 3.25 & $\ldots$ & $1.54 \mathrm{~A}$ \\
\hline Fe II $\lambda 2.061$ & $\ldots$ & 4.80 & $\ldots$ & 2.89 & $\ldots$ & $\ldots$ \\
\hline$? \lambda 2.068$ & $\ldots$ & $\ldots$ & $\ldots$ & $\ldots$ & $\ldots$ & $0.24 \mathrm{~A}$ \\
\hline Fe II $\lambda 2.091$ & $\ldots$ & 15.81 & 0.34 & 9.22 & $\ldots$ & $\ldots$ \\
\hline$? \lambda 2.097$ & $\ldots$ & 0.62 & $\ldots$ & $\ldots$ & $\ldots$ & $\ldots$ \\
\hline He I $\lambda 2.112 / 3$ & $\ldots$ & 0.17 & $\ldots$ & $2.03 \mathrm{~A}$ & $\ldots$ & $\ldots$ \\
\hline He I $\lambda 2.114$ & $\ldots$ & 0.15 & $\ldots$ & $\ldots$ & $\ldots$ & $\ldots$ \\
\hline ?N III/C III $\lambda 2.116$ & $\ldots$ & 0.38 & $\ldots$ & $\ldots$ & $\ldots$ & $\ldots$ \\
\hline$?[\mathrm{Fe}$ II] $] / \mathrm{Al}$ I $\lambda 2.118$ & $\ldots$ & 1.31 & $\ldots$ & 1.15 & $\ldots$ & $\ldots$ \\
\hline$? \mathrm{H}_{2} / \mathrm{Fe}$ II $\lambda 2.124$ & $\ldots$ & 0.41 & $\ldots$ & $\ldots$ & $\ldots$ & $\ldots$ \\
\hline [Fe II] $\lambda 2.133$ & $\ldots$ & 0.75 & $\ldots$ & 0.69 & $\ldots$ & $\ldots$ \\
\hline $\operatorname{Mg}$ II $\lambda 2.137$ & $\ldots$ & 3.99 & 0.19 & 2.14 & $\ldots$ & 0.30 \\
\hline Mg II $\lambda 2.144$ & $\ldots$ & 2.63 & 0.27 & 1.69 & $\ldots$ & 0.30 \\
\hline$?[\mathrm{Fe}$ II $] / \mathrm{He}$ I $\lambda 2.148$ & $\ldots$ & 0.76 & $\ldots$ & $\ldots$ & $\ldots$ & $\ldots$ \\
\hline Не I $\lambda 2.150$ & $\ldots$ & $\ldots$ & $\ldots$ & $0.56 \mathrm{~A}$ & $\ldots$ & $\ldots$ \\
\hline Не I $\lambda 2.161$ & $\ldots$ & 2.42 & $\ldots$ & $0.93 \mathrm{~A}$ & $\ldots$ & $\ldots$ \\
\hline H I Br $\gamma \lambda 2.166$ & $2.88 \mathrm{~A}$ & 84.51 & 0.60 & 13.78 & $2.93 \mathrm{~A}$ & $2.20 \mathrm{~A}$ \\
\hline Не I $\lambda 2.166$ & $\ldots$ & $\mathrm{Bl}$ & $\ldots$ & B1 & $\ldots$ & $\ldots$ \\
\hline$? \lambda 2.169$ & $\ldots$ & 2.96 & $\ldots$ & $\ldots$ & $\ldots$ & ... \\
\hline$? \lambda 2.174$ & $\ldots$ & 0.72 & $\ldots$ & 0.22 & $\ldots$ & $\ldots$ \\
\hline$? \lambda 2.187$ & $\ldots$ & 0.85 & $\ldots$ & ... & $\ldots$ & $\ldots$ \\
\hline $\mathrm{Na} I \lambda 2.206$ & 0.26 & 0.53 & $\ldots$ & $\ldots$ & 0.81 & 1.67 \\
\hline $\mathrm{Na}$ I $\lambda 2.209$ & 0.20 & 0.33 & $\ldots$ & $\ldots$ & 0.38 & 1.06 \\
\hline [Fe III] $\lambda 2.219$ & $\ldots$ & 0.42 & $\ldots$ & $\ldots$ & $\ldots$ & $\ldots$ \\
\hline$? \mathrm{H}_{2} \lambda 2.225$ & $\ldots$ & 0.60 & $\ldots$ & 0.62 & $\ldots$ & $\ldots$ \\
\hline ?He I $\lambda 2.228$ & $\ldots$ & 0.33 & $\ldots$ & $\ldots$ & $\ldots$ & $\ldots$ \\
\hline$?[\mathrm{Fe} \mathrm{II}] \lambda 2.238$ & $\ldots$ & 0.34 & $\ldots$ & $\ldots$ & $\ldots$ & $\ldots$ \\
\hline$?[\mathrm{Fe}$ II $] \lambda 2.241$ & $\ldots$ & 2.02 & $\ldots$ & 0.50 & $\ldots$ & $\ldots$ \\
\hline$[\mathrm{Fe}$ II] $] 2.254$ & $\ldots$ & ( & $\ldots$ & 0.58 & $\ldots$ & $\ldots$ \\
\hline ?He II $\lambda 2.258$ & $\ldots$ & 0.79 & $\ldots$ & 0.36 & $\ldots$ & $\ldots$ \\
\hline [Fe II] $\lambda 2.302$ & $\ldots$ & 0.33 & $\ldots$ & $\ldots$ & $\ldots$ & $\ldots$ \\
\hline$? \mathrm{He} \mathrm{I} /[\mathrm{Fe} \mathrm{II}] /[\mathrm{Ni} \mathrm{II}] \lambda 2.309$ & 1.02 & $\ldots$ & $\ldots$ & 0.56 & $\ldots$ & $\ldots$ \\
\hline $\mathrm{Fe}$ II/C III $\lambda 2.325$ & $\ldots$ & 0.37 & $\ldots$ & $\ldots$ & $\ldots$ & $\ldots$ \\
\hline
\end{tabular}

Notes. Measurements are in units of $\AA$ and for emission lines unless indicated by an A, indicating the line is in absorption. Bl indicates a blended line. 